Journal of Geophysical Research: Earth Surface

\author{
RESEARCH ARTICLE \\ 10.1002/2015JF003605 \\ Key Points: \\ - Macroscopic heat and vapor transfer \\ models in snow are derived by \\ homogenization \\ - Macroscopic effective properties and \\ source terms from phase change are \\ defined \\ - The effects of the air convection \\ on the macroscopic transfers are \\ highlighted
}

Supporting Information: - Text S1

Correspondence to: N. Calonne and C. Geindreau, neige.calonne@slf.ch; christian.geindreau@3sr-grenoble.fr

Citation:

Calonne, N., C. Geindreau, and F. Flin (2015), Macroscopic modeling of heat and water vapor transfer with phase change in dry snow based on an upscaling method: Influence of air convection, J. Geophys. Res. Earth Surface, 120, 2476-2497 doi:10.1002/2015JF003605.

Received 11 MAY 2015 Accepted 11 OCT 2015 Accepted article online 16 OCT 2015 Published online 8 DEC 2015

(O2015. American Geophysical Union. All Rights Reserved.

\section{Macroscopic modeling of heat and water vapor transfer with phase change in dry snow based on an upscaling method: Influence of air convection}

\author{
N. Calonne ${ }^{1,2,3,4}$, C. Geindreau ${ }^{2,3}$, and F. Flin ${ }^{1}$ \\ ${ }^{1}$ Météo-France-CNRS, CNRM-GAME UMR 3589, CEN, Saint Martin d'Hères, France, ${ }^{2} 3 S R$ Laboratory, University of \\ Grenoble Alpes, Grenoble, France, ${ }^{3}$ CNRS, Grenoble, France, ${ }^{4}$ Now at WSL Institute for Snow and Avalanche Research SLF, \\ Davos Dorf, Switzerland
}

Abstract At the microscopic scale, i.e., pore scale, dry snow metamorphism is mainly driven by the heat and water vapor transfer and the sublimation-deposition process at the ice-air interface. Up to now, the description of these phenomena at the macroscopic scale, i.e., snow layer scale, in the snowpack models has been proposed in a phenomenological way. Here we used an upscaling method, namely, the homogenization of multiple-scale expansions, to derive theoretically the macroscopic equivalent modeling of heat and vapor transfer through a snow layer from the physics at the pore scale. The physical phenomena under consideration are steady state air flow, heat transfer by conduction and convection, water vapor transfer by diffusion and convection, and phase change (sublimation and deposition). We derived three different macroscopic models depending on the intensity of the air flow considered at the pore scale, i.e., on the order of magnitude of the pore Reynolds number and the Péclet numbers: (A) pure diffusion, (B) diffusion and moderate convection (Darcy's law), and (C) strong convection (nonlinear flow). The formulation of the models includes the exact expression of the macroscopic properties (effective thermal conductivity, effective vapor diffusion coefficient, and intrinsic permeability) and of the macroscopic source terms of heat and vapor arising from the phase change at the pore scale. Such definitions can be used to compute macroscopic snow properties from 3-D descriptions of snow microstructures. Finally, we illustrated the precision and the robustness of the proposed macroscopic models through 2-D numerical simulations.

\section{Introduction}

At the microscopic scale, i.e., at the grains or pore scale, dry snow metamorphism is mainly driven by the heat and water vapor transfer and the sublimation-deposition process at the ice/air interface. The snowpack models [Brun et al., 1989; Jordan, 1991; Lehning et al., 2002a, 2006; Vionnet et al., 2012], which are used for various applications as avalanche forecasts or climate studies, require the description of these phenomena at the macroscopic scale, i.e., at the snow layer scale. Up to now, such models have been developed using a phenomenological approach. They can differ in their descriptions of the macroscopic heat and vapor transfer (vapor diffusion, convection effects, etc.), in their definitions of the effective properties (effective thermal conductivity, effective vapor diffusion, etc.), and also in their macroscopic source terms of heat and vapor, which reflect the effects of the phase change at the pore scale.

At the microscopic scale, air convection can occur through the pore spaces of snow and is classically divided into two types: forced convection and natural convection. Air convection in snow is generated by the wind at the surface of the snowpack that creates a "wind-pumping ventilation" across the air pores of the upper snow layers [Albert and McGilvary, 1992; Albert and Shultz, 2002]. Natural convection is not generated by an external source but simply by the differences in air density caused by the local temperature gradients in snow. This mechanism seems mainly influent for the snow layers that show large pores [Sturm and Johnson, 1991]. Both convections contribute to the heat and vapor transfer across the pores and might significantly impact the temperature and vapor density field through the snow layers [e.g., Akitaya, 1974; Powers et al., 1985; Brun and Touvier, 1987; Sokratov and Sato, 2000; Albert et al., 2004].

Based on a phenomenological model, Albert and McGilvary [1992] investigated the thermal effects due to air flow and vapor transport in dry snow. Within this model, the air flow is described by the classical Darcy's 
law, whereas the heat and water vapor transfer is described by two coupled equations, including convection effects and a source term induced by the phase change. Their source term depends on a mass transfer coefficient, defined as proportional to the air flow intensity through the pore Reynolds number [Albert and McGilvary, 1992; Neumann et al., 2009]. They concluded that for applications concerned only with temperature predictions, the forced convection should be considered for the heat transport but vapor transport may be ignored. To our knowledge, the snowpack model SNOWPACK [Lehning et al., 2002b] is the only one that attempts to take into account the effect of wind pumping on the temperature distribution of the surface snow layers. For that purpose, they introduced a "diffusivity due to wind pumping" into the parametrization of the effective thermal conductivity.

In Calonne et al. [2014a], we recently derived the macroscopic models for the heat and water vapor transfer with phase change, but neglecting the air convection, from the physics at the pore scale using an upscaling method, namely, the homogenization of multiple-scale expansions [Bensoussan et al., 1978; Sanchez-Palencia, 1980; Auriault, 1991; Geindreau and Auriault, 2001; Auriault et al., 2009]. Here we propose to investigate the influence of the forced air convection, typically induced by wind pumping, on these macroscopic models by following the same methodology. Besides providing the theoretical expression of all terms of the macroscopic model (effective properties, source terms, etc.), the idea is to investigate the relative impact of the convective transport on the temperature and vapor density profile of snow layers depending on the physics considered at the pore scale. The first part of the paper is dedicated to a step-by-step description of the homogenization method, starting from the physical description of the heat and vapor transfer with phase change at the pore scale to the derivation of the macroscopic equivalent models. Three main cases of macroscopic models are obtained and discussed. The second part consists in 2-D numerical simulations, which were performed on a simple geometry to illustrate the proposed macroscopic models and to evaluate their precision and robustness.

\section{Derivation of the Macroscopic Modeling}

\subsection{Homogenization Method}

Physical phenomena in heterogeneous media such as snow can be homogenized, i.e., they can be modeled by a continuous macroscopic equivalent description, if the condition of separation of scales is satisfied [Bensoussan et al., 1978; Sanchez-Palencia, 1980; Auriault, 1991; Auriault et al., 2009]. This fundamental condition may be expressed as $\varepsilon=I / L \ll 1$ where $I$ and $L$ are the characteristic lengths of the heterogeneities at the pore scale and of the macroscopic sample or excitation, respectively. This condition implies the existence of a representative elementary volume (REV) of size I of both the material and the excitation. In the following, the macroscopic equivalent model is obtained from the description at the heterogeneity scale, i.e., the REV scale, by using the homogenization technique of multiple-scale expansions [Bensoussan et al., 1978; Sanchez-Palencia, 1980] and by following the methodology presented in Auriault [1991]. More precisely, the macroscopic equivalent modeling of heat and water vapor transfer through a snow layer is obtained from the description of the physics at the pore scale by the following: (i) assuming the medium to be periodic, without loss of generality since the condition of separation of scales is fulfilled [Auriault, 2011]; (ii) writing the local description in a dimensionless form; (iii) evaluating the obtained dimensionless numbers with respect to the coefficient of separation of scales $\varepsilon$; (iv) looking for the unknown fields in the form of asymptotic expansions in powers of $\varepsilon$; and $(v)$ solving the successive boundary value problems that are obtained after introducing these expansions in the local dimensionless description. The macroscopic equivalent model is obtained from compatibility conditions which are the necessary conditions for the existence of solutions to the boundary value problems.

\subsection{Description of the Physics at the Microscopic Scale}

Let us assume that a snow layer can be represented by a collection of spatially periodic REVs with a characteristic length $I$, such that the scale parameter $\varepsilon=I / L \ll 1$ (Figure 1 ). Within the REV of snow $\Omega$, the domains occupied by ice and air are denoted by $\Omega_{i}$ and $\Omega_{a}$, respectively. The interface of ice is denoted by $\Gamma, \mathbf{n}^{\Gamma}$ is the outward unit normal vector of $\Omega_{i}$, and $\mathbf{t}^{\boldsymbol{\Gamma}}$ is the tangential unit normal vector at the interface. Throughout the paper, the subscripts $i$ and $a$ are related to quantities defined in $\Omega_{i}$ and $\Omega_{a}$, respectively. The air velocity in the pores of snow is typically lower than $0.1 \mathrm{~m} \mathrm{~s}^{-1}$ [Albert and McGilvary, 1992; Albert, 1993], and thus the Mach number, defined as the ratio of the air velocity over the sound velocity, is much smaller than 0.3 . Consequently, at the pore scale, the air flow is assumed to be incompressible and is described by the Navier-Stokes equation. The phenomena considered at microscale are (i) the steady state air flow within the pores 


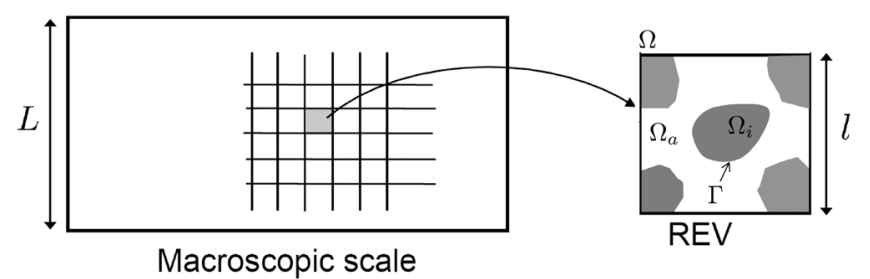

Figure 1. Macroscopic sample, i.e., a snow layer, and representative elementary volume (REV) with period $\Omega$.

(equations (1) and (2)), (ii) the heat transfer by diffusion in ice and by diffusion-convection in air (equations (3) and (4)), (iii) the water vapor transfer by diffusion-convection in air (equation (5)), and (vi) the phase change at the ice-pore interface (sublimation of ice and deposition of vapor), i.e., the growth or decay of ice, (equations (6), (9), and (10)). Assuming that the properties of air and ice are isotropic, the above physical phenomena at the pore scale are described by the following set of balance equations:

$$
\begin{gathered}
\rho_{a} \mathbf{v}_{a} \cdot \operatorname{grad} \mathbf{v}_{a}=\operatorname{div}\left(\mu_{a} \operatorname{grad} \mathbf{v}_{a}\right)-\operatorname{grad} p_{a} \quad \text { in } \Omega_{a} \\
\operatorname{div} \mathbf{v}_{a}=0 \quad \text { in } \Omega_{a} \\
\rho_{i} C_{i} \frac{\partial T_{i}}{\partial t}-\operatorname{div}\left(\kappa_{i} \operatorname{grad} T_{i}\right)=0 \quad \text { in } \Omega_{i} \\
\rho_{a} C_{a} \frac{\partial T_{a}}{\partial t}+\rho_{a} C_{a} \mathbf{v}_{a} \cdot \operatorname{grad} T_{a}-\operatorname{div}\left(\kappa_{a} \operatorname{grad} T_{a}\right)=0 \quad \text { in } \Omega_{a} \\
\frac{\partial \rho_{v}}{\partial t}+\mathbf{v}_{a} \cdot \operatorname{grad} \rho_{v}-\operatorname{div}\left(D_{v} \operatorname{grad} \rho_{v}\right)=0 \quad \text { in } \Omega_{a}
\end{gathered}
$$

This set of balance equations must be completed on the ice-air interface $(\Gamma)$ by the following conditions taking into account the phase change:

1. The continuity of mass fluxes

$$
\rho_{a}\left(\mathbf{v}_{\mathbf{a}}-\mathbf{w}\right) \cdot \mathbf{n}^{\boldsymbol{\Gamma}}=-\rho_{i} \mathbf{w} \cdot \mathbf{n}^{\boldsymbol{\Gamma}} \text { on } \Gamma
$$

2. The continuity of tangential velocities (no-slip condition)

$$
\mathbf{v}_{\mathbf{a}} \cdot \mathbf{t}^{\mathbf{\Gamma}}=0 \text { on } \Gamma
$$

3. The continuity of temperatures

$$
T_{i}=T_{a} \quad \text { on } \Gamma
$$

4. The continuity of heat fluxes

$$
\kappa_{i} \operatorname{grad} T_{i} \cdot \mathbf{n}^{\Gamma}-\kappa_{a} \operatorname{grad} T_{a} \cdot \mathbf{n}^{\Gamma}=L_{s g} \mathbf{w} \cdot \mathbf{n}^{\Gamma} \quad \text { on } \Gamma
$$

5. The continuity of water vapor fluxes

$$
D_{v} \operatorname{grad} \rho_{v} \cdot \mathbf{n}^{\Gamma}=\left(\rho_{i}-\rho_{v}\right) \mathbf{w} \cdot \mathbf{n}^{\Gamma} \quad \text { on } \Gamma
$$

In the above set of equations, $t$ is the time (s), $\mathbf{v}_{a}$ is the air velocity $\left(\mathrm{m} \mathrm{s}^{-1}\right), p_{a}$ is the air pressure $(\mathrm{Pa}), \mu_{a}$ is the air dynamic viscosity $\left(\mathrm{Pa} \mathrm{s}^{-1}\right), T$ is the temperature $(\mathrm{K}), \kappa$ is the thermal conductivity $\left(\mathrm{W} \mathrm{m}^{-1} \mathrm{~K}^{-1}\right), \rho$ is the density $\left(\mathrm{kg} \mathrm{m}^{-3}\right), C$ is the specific heat capacity $\left(\mathrm{J} \mathrm{kg}^{-1} \mathrm{~K}^{-1}\right), L_{s g}$ is the latent heat of sublimation $\left(\mathrm{J} \mathrm{m}^{-3}\right), \mathbf{w}$ is the interface growth velocity $\left(\mathrm{m} \mathrm{s}^{-1}\right), \rho_{v}$ is the partial density of water vapor in air $\left(\mathrm{kg} \mathrm{m}^{-3}\right), D_{v}$ is the water vapor diffusion coefficient in air $\left(\mathrm{m}^{2} \mathrm{~s}^{-1}\right)$, and div and grad are the divergence and gradient operators with respect to the physical space variable $\mathbf{X}$, respectively. 
At the interface, the heat and vapor transfer is coupled through the normal interface growth velocity $w_{n}=\mathbf{w} \cdot \mathbf{n}^{\Gamma}$ (equations (9) and (10)), which is given by the Hertz-Knudsen equation:

$$
w_{n}=\mathbf{w} \cdot \mathbf{n}^{\Gamma}=\frac{1}{\beta}\left[\frac{\rho_{v}-\rho_{v s}\left(T_{a}\right)}{\rho_{v s}\left(T_{a}\right)}-d_{0} K\right] \text { on } \Gamma
$$

where $\beta$ is the interface kinetic coefficient $\left(\mathrm{s} \mathrm{m}^{-1}\right), \rho_{v s}$ is the saturation water vapor density in air $\left(\mathrm{kg} \mathrm{m}^{-3}\right), d_{0}$ is the capillary length $(\mathrm{m})$, and $K$ is the interface mean curvature $\left(\mathrm{m}^{-1}\right)$. Let us remark that the interface kinetic coefficient $\beta$ is linked to the deposition coefficient $\alpha$ by

$$
\beta=\frac{1}{\alpha} \frac{\rho_{i}}{\rho_{v s}\left(T_{a}\right)} \sqrt{\frac{2 \pi m}{k T_{a}}}
$$

where $m$ is the mass of a water molecule $(\mathrm{kg})$ and $k$ is the Boltzmann's constant equal to $1.38 \times 10^{-23} \mathrm{~J} \mathrm{~K}^{-1}$. The value of $w_{n}$ is positive when the ice surface grows and negative when the ice surface sublimates. The saturation vapor density $\rho_{v s}$ is given by the Clausius-Clapeyron's law:

$$
\rho_{v s}\left(T_{a}\right)=\rho_{v s}^{\text {ref }}\left(T^{\text {ref }}\right) \exp \left[\frac{L_{s g} m}{\rho_{i} k}\left(\frac{1}{T^{\text {ref }}}-\frac{1}{T_{a}}\right)\right]
$$

The reference values $T^{\text {ref }}$ and $\rho_{v s}^{\text {ref }}\left(T^{\text {ref }}\right)$ are equal here to $273 \mathrm{~K}$ and $2.173 \times 10^{-3} \mathrm{~kg} \mathrm{~m}^{-3}$, respectively. In what follows, for the sake of simplicity, we will suppose that all material properties $\left(\mu_{a}, \rho, C, \kappa, D_{v}, \beta\right.$, and $m$ ) do not depend on the temperature, except for the density of water vapor that is calculated using the Clausius-Clapeyron' law (12).

\subsection{Dimensionless Pore-Scale Description: Normalization}

Following the methodology presented in Auriault [1991], we introduce in the set of equations (1)-(10) the following representation of all dimensional variables $\varphi: \varphi=\varphi_{c} \varphi^{*}$. The subscript $c$ denotes characteristic quantities (constant), and the superscript asterisk denotes the dimensionless microscopic variables. Consequently, the formal dimensionless set of equations that describes the physics at the pore scale is written

$$
\begin{aligned}
& {[R e] \rho_{a}^{*} \mathbf{v}_{\mathbf{a}}{ }^{*} \cdot \operatorname{grad}^{*} \mathbf{v}_{\mathbf{a}}{ }^{*}=\operatorname{div}^{*}\left(\mu_{a}^{*} \operatorname{grad}^{*} \mathbf{v}_{\mathbf{a}}{ }^{*}\right)-[Q] \operatorname{grad}^{*} p_{a}^{*} \text { in } \Omega_{a}} \\
& \operatorname{div}^{*} \mathbf{v}_{\mathbf{a}}{ }^{*}=0 \quad \text { in } \Omega_{a} \\
& {\left[F_{i}^{\top}\right] \rho_{i}^{*} C_{i}^{*} \frac{\partial T_{i}^{*}}{\partial t^{*}}-\operatorname{div}^{*}\left(\kappa_{i}^{*} \operatorname{grad}^{*} T_{i}^{*}\right)=0 \quad \text { in } \Omega_{i}} \\
& {\left[F_{a}^{\top}\right] \rho_{a}^{*} C_{a}^{*} \frac{\partial T_{a}^{*}}{\partial t^{*}}+\left[P e^{\top}\right] \rho_{a}^{*} C_{a}^{*} \mathbf{v}_{\mathbf{a}}^{*} \cdot \operatorname{grad}^{*} T_{a}^{*}-\operatorname{div}^{*}\left(\kappa_{a}^{*} \operatorname{grad}^{*} T_{a}^{*}\right)=0 \quad \text { in } \Omega_{a}} \\
& {\left[F_{a}^{\rho}\right] \frac{\partial \rho_{v}^{*}}{\partial t^{*}}+\left[P e^{\rho}\right] \mathbf{v}_{\mathbf{a}}^{*} \cdot \operatorname{grad}^{*} \rho_{v}^{*}-\operatorname{div}^{*}\left(D_{v}^{*} \operatorname{grad}^{*} \rho_{v}^{*}\right)=0 \text { in } \Omega_{a}} \\
& \rho_{a}^{*}\left(\mathbf{v}_{\mathbf{a}}^{*}-[M] \mathbf{w}^{*}\right) \cdot \mathbf{n}^{\Gamma}=-[N] \rho_{i}^{*} \mathbf{w}^{*} \cdot \mathbf{n}^{\Gamma} \quad \text { on } \Gamma \\
& \mathbf{v}_{\mathbf{a}}^{*} \cdot \mathbf{t}^{\Gamma}=0 \text { on } \Gamma \\
& T_{i}^{*}=T_{a}^{*} \quad \text { on } \Gamma \\
& {[K] \kappa_{i}^{*} \operatorname{grad}^{*} T_{i}^{*} \cdot \mathbf{n}^{\Gamma}-\kappa_{a}^{*} \operatorname{grad}^{*} T_{a}^{*} \cdot \mathbf{n}^{\Gamma}=[H] L_{s g}^{*} \mathbf{w}^{*} \cdot \mathbf{n}^{\Gamma} \quad \text { on } \Gamma} \\
& D_{v}^{*} \operatorname{grad}^{*} \rho_{v}^{*} \cdot \mathbf{n}^{\Gamma}=[W] \rho_{i}^{*} \mathbf{w}^{*} \cdot \mathbf{n}^{\Gamma} \text { on } \Gamma
\end{aligned}
$$


This dimensionless pore scale description introduces 12 dimensionless numbers which characterize the intensity of the physical phenomena at the pore scale. These dimensionless numbers are defined as follows:

$$
\begin{aligned}
& {[R e]=\frac{v_{a_{c}} \rho_{a_{c}}}{\mu_{a_{c}}}, \quad[Q]=\frac{p_{a_{c}} l}{\mu_{a_{c}} v_{a_{c}}},} \\
& {\left[F_{i}^{\top}\right]=\frac{P^{2} \rho_{i_{c}} C_{i_{c}}}{t_{c} \kappa_{i_{c}}}, \quad\left[F_{a}^{\top}\right]=\frac{P^{2} \rho_{a_{c}} C_{a_{c}}}{t_{c} \kappa_{a_{c}}}, \quad\left[F_{a}^{\rho}\right]=\frac{P^{2}}{D_{v_{c}} t_{c}},} \\
& {\left[P e^{\top}\right]=\frac{l \rho_{a_{c}} C_{a_{c}} v_{a_{c}}}{\kappa_{a_{c}}}=[\operatorname{Pr}][\operatorname{Re}], \quad\left[P e^{\rho}\right]=\frac{v_{a_{c}} l}{D_{v_{c}}}=[S c][R e],} \\
& {[M]=\frac{w_{n_{c}}}{v_{a_{c}}}, \quad[N]=\frac{\rho_{i_{c}}}{\rho_{a_{c}}}} \\
& {[K]=\frac{\kappa_{i_{c}}}{\kappa_{a_{c}}}, \quad[H]=\frac{I L_{s g_{c}} W_{n_{c}}}{\kappa_{a_{c}} T_{a_{c}}} \text {, and }[W]=\frac{I \rho_{i_{c}} W_{n_{c}}}{D_{v_{c}} \rho_{v_{c}}} \text {. }}
\end{aligned}
$$

In the above set of equations, $[R e]$ is the classical pore Reynolds number that characterizes the intensity of the flow in $\Omega_{a}$, and $[Q]$ is a dimensionless number that measures the ratio between the pressure and the viscous stress in $\Omega_{a}$. The dimensionless numbers $\left[F_{i}^{\top}\right]$ and $\left[F_{a}^{\top}\right]$ correspond to the inverses of the Fourier numbers in $\Omega_{i}$ and $\Omega_{a}$, respectively, and $\left[F_{a}^{\rho}\right]$ is an analogous inverse Fourier number for the transient water vapor transfer by diffusion in $\Omega_{a}$. Conceptually, they measure the ratio of the quantity (heat or vapor) storage rate by the diffusive transport rate. $\left[\mathrm{Pe}^{\top}\right]$ and $\left[\mathrm{Pe}^{\rho}\right]$ are the Péclet numbers associated to the thermal and water vapor transfer in $\Omega_{a}$. They measure the ratio between the transfer by convection over the transfer by conduction or diffusion. Let us remark that $\left[\mathrm{Pe}^{\top}\right]$ can be expressed as the product of the Reynolds number $[R e]$ and the Prandtl number $[\mathrm{Pr}]$ equal to $\left(\mu_{a_{c}} C_{a_{c}}\right) / \kappa_{a_{c}} \sim 0.71 \mathrm{in} \Omega_{a}$. Similarly, $\left[\mathrm{Pe} e^{\rho}\right]$ is written as the product of the Reynolds number $[R e]$ and the Schmidt number [Sc] equal to $\mu_{a_{c}} /\left(\rho_{a_{c}} D_{v_{c}}\right) \sim 0.63$ in $\Omega_{a}$. Dimensionless numbers [M], $[N],[K],[H]$, and $[W]$ characterize the physical phenomena at the ice-air interface. In particular, $[M]$ and $[N]$ characterize the continuity of the mass fluxes at the ice-air interface. $[H]$ characterizes the ratio between the heat flux induced by phase changes and the heat flux from heat diffusion in the air phase. Similarly, $[W]$ is defined as the ratio between the vapor flux from phase changes and the vapor flux from vapor diffusion in the air phase.

\subsection{Estimation of the Dimensionless Numbers}

The next step of the homogenization process consists in estimating the above 12 dimensionless numbers with respect to the scale parameter $\varepsilon=I / L$. In practice, $I$ and $L$ correspond to the order of magnitude of the size of the REV and the thickness of the snow layer, respectively. For the following estimations, we assumed that $I \approx 5.10^{-4} \mathrm{~m}$ and $L \approx 0.1 \mathrm{~m}$, leading to $\varepsilon=5.10^{-3}$. The characteristic time $t_{c}$ is the time over which we intend to describe the heat and water vapor transfer through the snowpack, i.e., the characteristic time of observation. As in Auriault and Adler [1995] and Auriault et al. [2009], we can introduce two macroscopic characteristic times $t^{\mathrm{di}}$ and $t^{\mathrm{co}}$ associated to the diffusion and the convection phenomena, respectively. They are defined as follows:

$$
\begin{aligned}
t^{\mathrm{di}}=\mathcal{O}\left(L^{2} / \alpha_{i_{c}}\right) & =\mathcal{O}\left(L^{2} / \alpha_{i_{a}}\right)=\mathcal{O}\left(L^{2} / D_{v_{c}}\right) \\
t^{\mathrm{Co}} & =\mathcal{O}\left(L / v_{a_{c}}\right)
\end{aligned}
$$

where $\alpha_{i_{c}}=\kappa_{i_{c}} /\left(C_{i_{c}} \rho_{i_{c}}\right)$ and $\alpha_{a_{c}}=\kappa_{a_{c}} /\left(C_{a_{c}} \rho_{a_{c}}\right)$ are the thermal diffusivity of the ice and the air phase, respectively. Using these definitions, the Péclet numbers $\left[P e^{\top}\right]$ and $\left[P e^{\rho}\right]$ can be rewritten as follows:

$$
\left[P e^{T}\right]=\left[P e^{\rho}\right]=\mathcal{O}\left(\varepsilon t^{\mathrm{co}} / t^{\mathrm{di}}\right)
$$

All the characteristic values of each variable arising in the dimensionless numbers (23) -(27) are summarized in Table 1 . The time $t_{c}$, the air velocity $v_{a_{c}}$, and the normal interface velocity $w_{n_{c}}$ are likely to vary, and different estimations are considered in the following depending on their values. In what follows, the dimensionless numbers are estimated in terms of order of magnitude with respect to $\varepsilon$. The order of magnitude of $\varepsilon^{p}$ is defined such as $\mathcal{O}\left(\varepsilon^{p-1 / 2}\right) \geq \mathcal{O}\left(\varepsilon^{p}\right) \geq \mathcal{O}\left(\varepsilon^{p+1 / 2}\right)$, with $p=1,2$, 3, etc. 
Table 1. Characteristic Values of the Properties Evaluated at $-10^{\circ} \mathrm{C}$ From the Literature [Massman, 1998; Kaempfer and Plapp, 2009; Calonne et al., 2014a]

\begin{tabular}{lcc} 
Symbol & Description & Value \\
\hline$T_{i_{c}} T_{a_{c}}$ & Temperature of ice, air & $263 \mathrm{~K}$ \\
$\kappa_{i_{c}}$ & Heat conductivity of ice & $2.3 \mathrm{~W} \mathrm{~m}^{-1} \mathrm{~K}^{-1}$ \\
$\kappa_{a_{c}}$ & Heat conductivity of air & $0.024 \mathrm{~W} \mathrm{~m}^{-1} \mathrm{~K}^{-1}$ \\
$C_{i_{c}}$ & Specific heat capacity of ice & $2000 \mathrm{~J} \mathrm{~kg}^{-1} \mathrm{~K}^{-1}$ \\
$C_{a_{c}}$ & Specific heat capacity of air & $1005 \mathrm{~J} \mathrm{~kg}^{-1} \mathrm{~K}^{-1}$ \\
$L_{s g_{c}}$ & Latent heat of sublimation of ice & $2.60 \times 10^{9} \mathrm{~J} \mathrm{~m}^{-3}$ \\
$w_{n_{c}}$ & Normal interface velocity & $10^{-14} \mathrm{to}^{-8} \mathrm{~m}^{-8} \mathrm{~m} \mathrm{~s}^{-1}$ \\
$D_{v_{c}}$ & Water vapor diffusion coefficient in air & $2.036 \times 10^{-5} \mathrm{~m}^{2} \mathrm{~s}^{-1}$ \\
$\rho_{v_{c}}$ & Water vapor density in air & $0.002 \mathrm{~kg} \mathrm{~m}^{-3}$ \\
$\rho_{i_{c}}$ & Ice density & $917 \mathrm{~kg} \mathrm{~m}^{-3}$ \\
$\rho_{a_{c}}$ & Air density & $1.335 \mathrm{~kg} \mathrm{~m}^{-3}$ \\
$I$ & Microscopic length & $5 \times 10^{-4} \mathrm{~m}$ \\
$L$ & Macroscopic length & $0.1 \mathrm{~m}$ \\
$\mu_{a_{c}}$ & Dynamic viscosity of air & $1.7 \times 10^{-5} \mathrm{~Pa} \mathrm{~s}$ \\
$p_{a_{c}}$ & Air pressure & $1.01325 \times 10^{5} \mathrm{~Pa}$ \\
$v_{a_{c}}$ & Air velocity & $10^{-8}$ to $10^{-3} \mathrm{~m} \mathrm{~s}^{-1}$ \\
$t_{c}$ & Time & see section 2.4 \\
\hline
\end{tabular}

Dimensionless Numbers Depending on $t_{c}$ and $v_{a_{c}}$. The inverses of Fourier numbers $\left[F_{i}^{\top}\right],\left[F_{a}^{\top}\right]$, and $\left[F_{a}^{\rho}\right]$ depend on the order of the characteristic time $t_{c}$, which can be the characteristic time of diffusion or convection. The characteristic time of convection is related to the characteristic intensity of the air flow $v_{a_{c}}$ (equation (29)). The Péclet numbers $\left[\mathrm{Pe}^{\top}\right]$ and $\left[\mathrm{Pe}^{\rho}\right]$ and the pore Reynolds number $[R e]$ are of the same order of magnitude (since the Prandtl and Schmidt numbers are both of the order of 1 ) and also depend on $v_{a_{c}}$. The characteristic value $v_{a_{c}}$ corresponds to an averaged value of the air velocity over the $\operatorname{REV}\left\langle v_{a}\right\rangle$. During wind pumping, the air velocity within a snow layer varies typically between $10^{-7}$ and $10^{-1} \mathrm{~m} \mathrm{~s}^{-1}$ [Albert and McGilvary, 1992; Albert, 1993]. Taking into account these results, Figure 2 shows that $[R e],\left[P e^{\top}\right]$, and $\left[\mathrm{Pe}^{\rho}\right]$ can take different orders of magnitude according to these values of $v_{a_{c}}$. Consequently, three cases can be considered:

1. Case A: when $v_{a_{c}} \leq 10^{-5} \mathrm{~m} \mathrm{~s}^{-1}$, diffusion dominates at the macroscopic scale and $t^{\mathrm{di}}=\varepsilon t^{\mathrm{co}}\left(t^{\mathrm{di}} \ll t^{\mathrm{co}}\right)$. To investigate this case, we use $t_{c}=t^{\mathrm{di}}$ as our characteristic time. This means that $\left[F_{i}^{\top}\right]=\left[F_{a}^{\top}\right]=\left[F_{a}^{\rho}\right]=\mathcal{O}\left(\varepsilon^{2}\right)$ and $[R e]=\left[P e^{\top}\right]=\left[P e^{\rho}\right] \leq \mathcal{O}\left(\varepsilon^{2}\right)$.

2. Case $\mathrm{B}$ : when $10^{-5} \mathrm{~m} \mathrm{~s}^{-1} \leq v_{a_{c}} \leq 10^{-3} \mathrm{~m} \mathrm{~s}^{-1}$, diffusion and convection are the same order of magnitude at the macroscopic scale and $t^{\mathrm{di}}=t^{\mathrm{co}}$. To investigate this case, we use $t_{c}=t^{\mathrm{di}}=t^{\mathrm{co}}$ as our characteristic time. This means that $\left[F_{i}^{\top}\right]=\left[F_{a}^{\top}\right]=\left[F_{a}^{\rho}\right]=\mathcal{O}\left(\varepsilon^{2}\right)$ and $[R e]=\left[P e^{\top}\right]=\left[P e^{\rho}\right]=\mathcal{O}(\varepsilon)$.

3. Case $C$ : when $10^{-3} \mathrm{~m} \mathrm{~s}^{-1} \leq v_{a_{c}} \leq 10^{-1} \mathrm{~m} \mathrm{~s}^{-1}$, convection dominates at the macroscopic scale and $t^{\mathrm{di}}=\varepsilon^{-1} t^{\mathrm{co}}$ $\left(t^{\mathrm{di}} \gg t^{\mathrm{co}}\right)$. To investigate this case, we use $t_{c}=t^{\mathrm{co}}$ as our characteristic time. This means that $\left[F_{i}^{\top}\right]=\left[F_{a}^{\top}\right]=$ $\left[F_{a}^{\rho}\right]=\mathcal{O}(\varepsilon)$ and $[R e]=\left[P e^{\top}\right]=\left[P e^{\rho}\right]=\mathcal{O}(1)$.

The fluid flow is also characterized by the dimensionless number [Q]. It can be shown [Auriault, 1991] by following a physical reasoning that $[Q]=\mathcal{O}\left(\varepsilon^{-1}\right)$, whatever the value of $v_{a_{c}}$.

Dimensionless Numbers Depending on $w_{n_{c}}$. The dimensionless numbers $[H]$ and $[W]$ depend on the intensity of the interface normal growth velocity $w_{n_{c}}$, which corresponds to an averaged value of the ice growth velocity over the REV $\left\langle w_{n}\right\rangle$. In practice, $w_{n}$ varies widely locally inside a REV, depending on the temperature gradient, temperature, and kinetic and curvature effects. Nevertheless, several experimental and numerical studies [Colbeck, 1983; Fukuzawa and Akitaya, 1993; Sturm et al., 1997; Kamata and Sato, 2007; Flin and Brzoska, 2008] have shown $w_{n}$ values ranging from 0 to $10^{-9} \mathrm{~m} \mathrm{~s}^{-1}$ for temperature gradients between 0 and $500 \mathrm{~K} \mathrm{~m}^{-1}$. Taking into account these results, Calonne et al. [2014a] have shown that $[H]$ and $[W]$ can take different orders of magnitude according to these values of $w_{n_{c}}$ : 


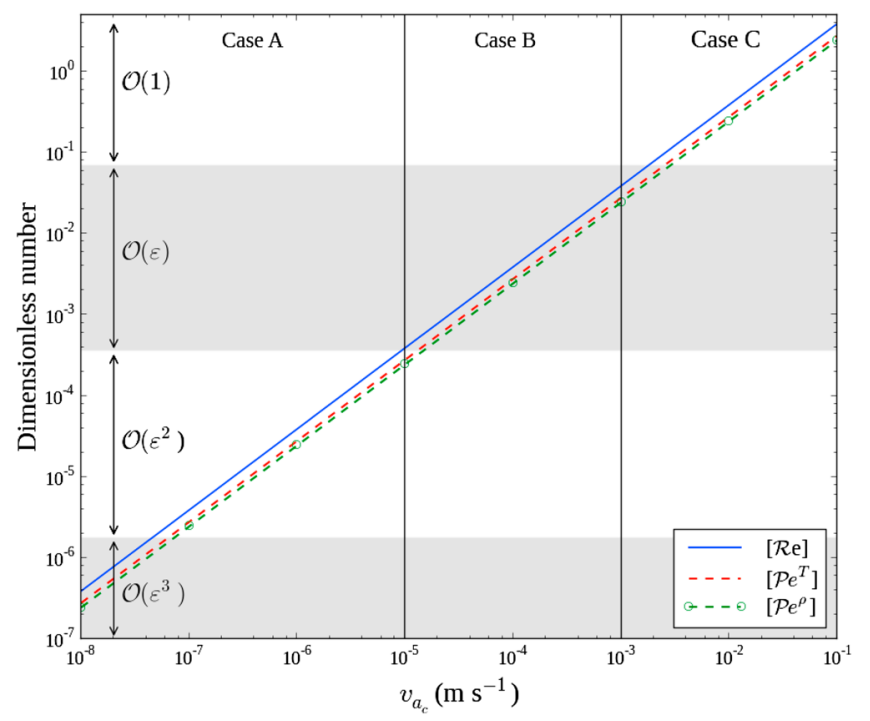

Figure 2. Estimation of dimensionless numbers $[R e],\left[P e^{\top}\right]$, and $\left[P e^{\rho}\right]$ versus the characteristic air velocity $v_{a_{c}}$ from characteristic values given in Table 1.

1. Case 0: when $w_{n_{c}} \approx 10^{-10} \mathrm{~m} \mathrm{~s}^{-1},[H]=\mathcal{O}\left(\varepsilon^{2}\right)$ and $[W]=\mathcal{O}(\varepsilon)$.

2. Case 1: when $w_{n_{c}} \approx 10^{-11} \mathrm{~m} \mathrm{~s}^{-1},[H]=\mathcal{O}\left(\varepsilon^{2}\right)$ and $[W]=\mathcal{O}\left(\varepsilon^{2}\right)$.

3. Case 2: when $w_{n_{c}} \approx 10^{-12} \mathrm{~m} \mathrm{~s}^{-1},[H]=\mathcal{O}\left(\varepsilon^{3}\right)$ and $[W]=\mathcal{O}\left(\varepsilon^{2}\right)$.

4. Case 3: when $w_{n_{c}} \leq 10^{-13} \mathrm{~m} \mathrm{~s}^{-1},[H] \leq \mathcal{O}\left(\varepsilon^{3}\right)$ and $[W] \leq \mathcal{O}\left(\varepsilon^{3}\right)$.

As in Calonne et al. [2014a], we will not investigate Case 0, which corresponds to the case of very large temperature gradients and is characterized by interfaces rapidly moving into vapor fields and significant latent heat effects.

Other Dimensionless Numbers. Finally, at the ice-pore interface, we have $[K]=\mathcal{O}(1)$ and $[N]=\mathcal{O}\left(\varepsilon^{-1}\right)$. The last dimensionless number $[M]$ is defined as the ratio of $w_{n_{c}}$ and $v_{a_{c}}$. Consequently, the order of magnitude of $[M]$ depends simultaneously on Cases A, B, and C and on Cases 1, 2, and 3 under consideration.

To conclude, nine different cases must be considered a priori. The order of magnitude of all the dimensionless numbers corresponding to these different cases are summarized in Figure 3. However, it can be shown that only three cases are of interest: Cases A1, B1, and C1. The other ones can be viewed as particular cases of these three main cases. Moreover, in Case $A 1$, when $[R e]=\left[P e^{\top}\right]=\left[P e^{\rho}\right] \leq \mathcal{O}\left(\varepsilon^{2}\right)$, the intensity of air flow is very small, and it can be shown that convection effects on the macroscopic heat and mass transfer are negligible. Thus, Case A1 corresponds to the case already studied by Calonne et al. [2014a], where the air convection is not taken into account. The Case B1 and Case C1 that correspond to the cases of moderate and strong convection, respectively, are investigated in the following.

\subsection{Asymptotic Analysis}

The next step is to introduce multiple-scale coordinates [Bensoussan et al., 1978; Sanchez-Palencia, 1980; Auriault, 1991]. The two characteristic lengths $L$ and $/$ introduce two dimensionless space variables, $\mathbf{x}^{*}=\mathbf{X} / L$ and $\mathbf{y}^{*}=\mathbf{X} / I$, where $\mathbf{X}$ is the physical space variable. The macroscopic (or slow) dimensionless space variable $\mathbf{x}^{*}$ is related to the microscopic (or fast) dimensionless space variable $\mathbf{y}^{*}$ by $\mathbf{x}^{*}=\varepsilon \mathbf{y}^{*}$. When $/$ is used as the characteristic length, the dimensionless derivative operator $\operatorname{grad}^{*}$ becomes $\left(\operatorname{grad}_{y^{*}}+\varepsilon \mathbf{g r a d}_{x^{*}}\right.$ ), where the subscripts $x^{*}$ and $y^{*}$ denote the derivatives with respect to the variables $\mathbf{x}^{*}$ and $\mathbf{y}^{*}$, respectively. Following the multiple-scale expansion technique [Bensoussan et al., 1978; Sanchez-Palencia, 1980; Auriault, 1991], the air velocity $\mathbf{v}_{\mathbf{a}}{ }^{*}$, the air pressure $p_{a^{\prime}}^{*}$ the ice temperature $T_{i}^{*}$, the air temperature $T_{a}^{*}$, and the water vapor $\rho_{v}^{*}$ are sought in the form of asymptotic expansions of powers of $\varepsilon$ :

$$
\varphi^{*}\left(\mathbf{x}^{*}, \mathbf{y}^{*}, t\right)=\varphi^{*(0)}\left(\mathbf{x}^{*}, \mathbf{y}^{*}, t\right)+\varepsilon \varphi^{*(1)}\left(\mathbf{x}^{*}, \mathbf{y}^{*}, t\right)+\varepsilon^{2} \varphi^{*(2)}\left(\mathbf{x}^{*}, \mathbf{y}^{*}, t\right)+\ldots
$$

where $\varphi^{*}=\mathbf{v}_{\mathbf{a}}{ }^{*}, p_{a}^{*}, T_{i}^{*}, T_{a}^{*}, \rho_{v}^{*}$ and the corresponding $\varphi^{*(i)}$ are periodic functions of period $\Omega$ with respect to the space variable $\mathbf{y}^{*}$. Substituting these expansions in the set (13)-(22) gives, by identification of like 


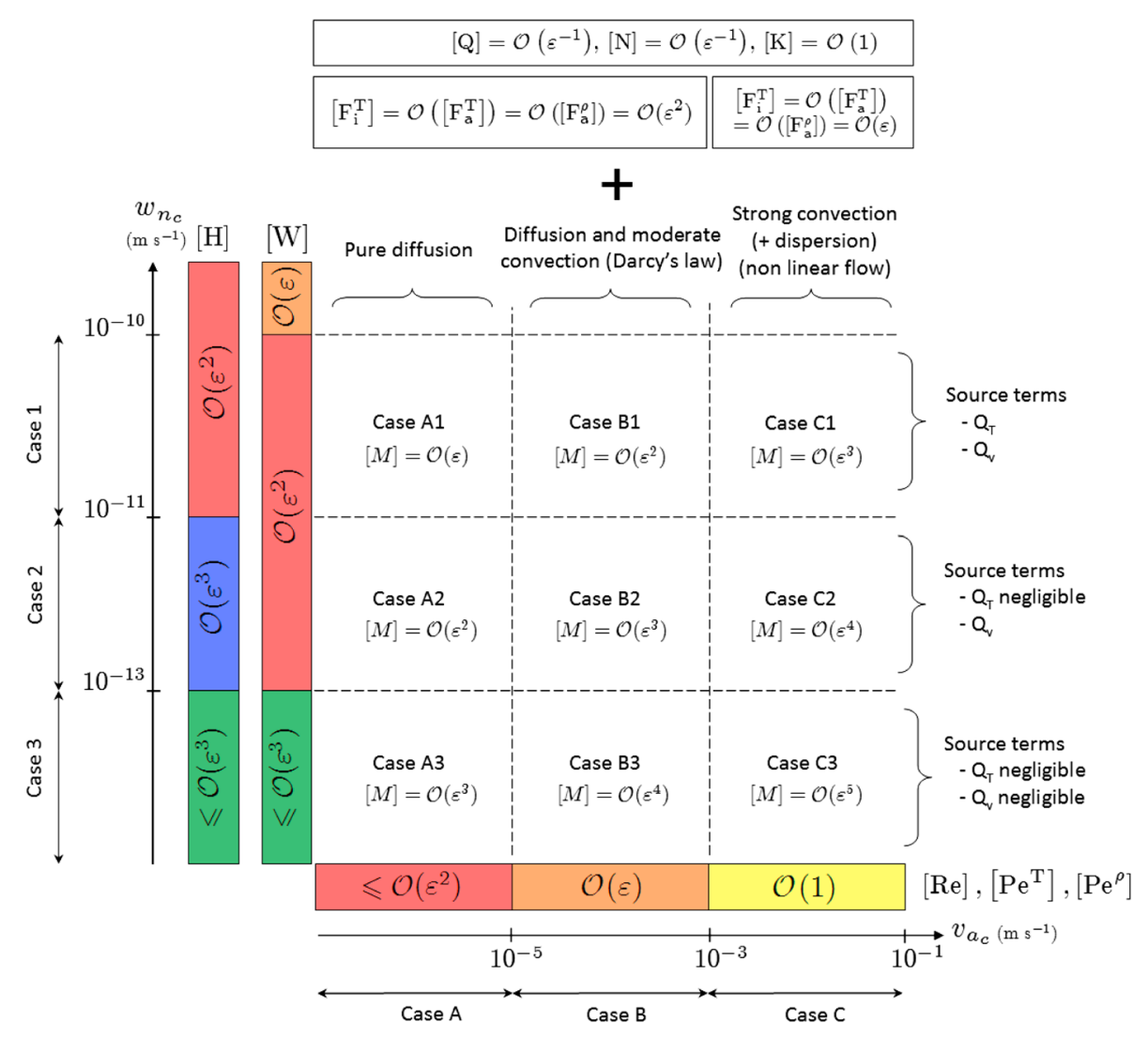

Figure 3. Synthesis of the different cases of dimensionless estimations, corresponding to different models, depending on the characteristic values considered in the description of the physics at the pore scale.

powers of $\varepsilon$, successive boundary value problems to be investigated. All the details concerning this asymptotic analysis in Case B1 and Case C1 are presented in the supporting information.

\subsection{Macroscopic Equivalent Descriptions}

According to the order of magnitude of the dimensionless numbers and based on the asymptotic analysis, we present here the derived equivalent macroscopic models at the first order of approximation $\left(T^{(0)}, \rho_{v}^{(0)}\right.$, etc.). Returning in dimensional variables, the heat and water vapor transfer through the snowpack is described by the following three main different macroscopic models (A1, B1, and C1). For a complete overview of all models, Case A1 presented in Calonne et al. [2014a] is recalled.

\subsubsection{Case $A 1$ (A2 and A3): Diffusion + Source Terms}

In Case A1, convection effects on the heat and water vapor transfer are negligible at the macroscopic scale. These transfers are thus purely diffusive and described by the following set of equations:

$$
\begin{gathered}
(\rho C)^{\text {eff }} \frac{\partial T^{(0)}}{\partial t}-\operatorname{div}\left(\mathbf{k}^{\text {eff }} \operatorname{grad} T^{(0)}\right)=\mathrm{SSA}_{V} L_{s g} w_{n}^{(0)} \\
\phi \frac{\partial \rho_{v}^{(0)}}{\partial t}-\operatorname{div}\left(\mathbf{D}^{\mathrm{eff}} \operatorname{grad} \rho_{v}^{(0)}\right)=-\mathrm{SSA}_{V} \rho_{i} w_{n}^{(0)}
\end{gathered}
$$

where $w_{n}^{(0)}$ is given by the Hertz-Knudsen equation (34) and the Clausius-Clapeyron's law (35) as follows:

$$
\begin{gathered}
w_{n}^{(0)}=\frac{1}{\beta}\left[\frac{\rho_{v}^{(0)}-\rho_{v s}^{(0)}\left(T^{(0)}\right)}{\rho_{v s}^{(0)}\left(T^{(0)}\right)}-d_{0} K\right] \\
\rho_{v s}^{(0)}\left(T^{(0)}\right)=\rho_{v s}^{\text {ref }} \exp \left[\frac{L_{s g} m}{\rho_{i} k}\left(\frac{1}{T^{\text {ref }}}-\frac{1}{T^{(0)}}\right)\right]
\end{gathered}
$$


and where $T^{(0)}$ is the macroscopic temperature, $\rho_{v}^{(0)}$ is the macroscopic vapor density in the air volume, $\phi$ is the porosity, $\mathrm{SSA}_{V}=|\Gamma| /|\Omega|$ is the specific surface area, $(\rho C)^{\text {eff }}$ is the effective thermal capacity, $\mathbf{k}^{\text {eff }}$ is the effective thermal conductivity tensor, and $\mathbf{D}^{\text {eff }}$ is the effective diffusion tensor. These effective properties are defined as follows:

$$
\begin{gathered}
(\rho C)^{\text {eff }}=(1-\phi) \rho_{i} C_{i}+\phi \rho_{a} C_{a} \\
\mathbf{k}^{\text {eff }}=\frac{1}{|\Omega|}\left(\int_{\Omega_{a}} \kappa_{a}\left(\mathbf{g r a d} \mathbf{t}_{a}+\mathbf{I}\right) \mathrm{d} \Omega+\int_{\Omega_{i}} \kappa_{i}\left(\mathbf{g r a d} \mathbf{t}_{i}+\mathbf{I}\right) \mathrm{d} \Omega\right) \\
\mathbf{D}^{\text {eff }}=\frac{1}{|\Omega|} \int_{\Omega_{a}} D_{v}\left(\mathbf{g r a d} \mathbf{g}_{v}+\mathbf{I}\right) \mathrm{d} \Omega
\end{gathered}
$$

where $\mathbf{t}_{a}$ and $\mathbf{t}_{i}$ are two periodic vectors solution of the following boundary value problem over the REV:

$$
\begin{gathered}
\operatorname{div}\left(\kappa_{i}\left(\operatorname{grad} \mathbf{t}_{i}+\mathbf{I}\right)\right)=0 \quad \text { in } \Omega_{i} \\
\operatorname{div}\left(\kappa_{a}\left(\operatorname{grad} \mathbf{t}_{a}+\mathbf{I}\right)\right)=0 \quad \text { in } \Omega_{a} \\
\mathbf{t}_{i}=\mathbf{t}_{a} \quad \text { on } \Gamma \\
\left(\kappa_{i}\left(\operatorname{grad} \mathbf{t}_{i}+\mathbf{I}\right)-\kappa_{a}\left(\operatorname{grad} \mathbf{t}_{a}+\mathbf{I}\right)\right) \cdot \mathbf{n}^{\Gamma}=0 \quad \text { on } \Gamma \\
\frac{1}{|\Omega|} \int_{\Omega}\left(\mathbf{t}_{a}+\mathbf{t}_{i}\right) \mathrm{d} \Omega=\mathbf{0}
\end{gathered}
$$

and where $\mathbf{g}_{v}$ is a periodic vector solution of the following boundary value problem over the REV:

$$
\begin{array}{cc}
\operatorname{div}\left(D_{v}\left(\operatorname{grad} \mathbf{g}_{v}+\mathbf{I}\right)\right)=0 & \text { in } \Omega_{a} \\
D_{v}\left(\operatorname{grad} \mathbf{g}_{v}+\mathbf{I}\right) \cdot \mathbf{n}^{\Gamma}=0 & \text { on } \Gamma \\
\frac{1}{|\Omega|} \int_{\Omega_{a}} \mathbf{g}_{v} \mathrm{~d} \Omega=\mathbf{0}
\end{array}
$$

In Case $\mathrm{A} 1$, the heat and water vapor transfer is described by two coupled equations of diffusion, including a source term of heat $Q_{T}=S S A_{V} L_{s g} w_{n}^{(0)}$, and a source term of vapor $Q_{v}=-S S A_{V} \rho_{i} w_{n}^{(0)}$. Let us remark that these source terms can also be written as $Q_{T}=L_{s g}(\mathrm{~d} \phi / \mathrm{d} t)$ and $Q_{v}=-\rho_{i}(\mathrm{~d} \phi / \mathrm{d} t)$, where $(\mathrm{d} \phi / \mathrm{d} t)$ represents the time derivative of the porosity. In Case $\mathrm{A} 2$, the source term $Q_{T}$ arising in the heat transfer equation is negligible. In Case $A 3$, the source terms $Q_{T}$ and $Q_{V}$ arising in both equations are negligible.

\subsubsection{Case B1 (B2 and B3): Diffusion and Moderate Convection + Source Terms}

In Case B1, the heat and water vapor transfer is governed by the diffusion and convection phenomena and described as follows:

$$
\begin{gathered}
\operatorname{div}_{x}\left\langle\mathbf{v}_{a}^{(0)}\right\rangle+\left(1-\frac{\rho_{i}}{\rho_{a}}\right) \operatorname{SSA}_{V} w_{n}^{(0)}=0 \\
\left\langle\mathbf{v}_{a}^{(0)}\right\rangle=-\frac{\mathbf{k}^{\mathrm{eff}}}{\mu_{a}} \mathbf{g r a d} p_{a}^{(0)} \\
(\rho C)^{\mathrm{eff}} \frac{\partial T^{(0)}}{\partial t}+\rho_{a} C_{a}\left\langle\mathbf{v}_{a}^{(0)}\right\rangle \cdot \operatorname{grad} T^{(0)}-\operatorname{div}\left(\mathbf{k}^{\mathrm{eff}} \mathbf{g r a d} T^{(0)}\right)=\mathrm{SSA}_{V} L_{s g} w_{n}^{(0)} \\
\phi \frac{\partial \rho_{v}^{(0)}}{\partial t}+\left\langle\mathbf{v}_{a}^{(0)}\right\rangle \cdot \operatorname{grad} \rho_{v}^{(0)}-\operatorname{div}\left(\mathbf{D}^{\mathrm{eff}} \mathbf{g r a d} \rho_{v}^{(0)}\right)=-\mathrm{SSA}_{V} \rho_{i} w_{n}^{(0)}
\end{gathered}
$$


where $\left\langle\mathbf{v}_{a}^{(0)}\right\rangle$ is the Darcy's velocity, and $\mathbf{K}^{\text {eff }}$ is the intrinsic permeability defined as follows:

$$
\mathbf{K}^{\text {eff }}=\frac{1}{|\Omega|} \int_{\Omega_{a}} \mathbf{k} \mathrm{d} \Omega
$$

where $\mathbf{k}$ is a periodic second order tensor, solution of the following boundary value problem over the REV (see equations (B21)-(B23) in the supporting information):

$$
\begin{gathered}
\operatorname{div}\left(\mu_{a} \operatorname{grad} \mathbf{k}\right)-\operatorname{grad} \mathbf{b}-\mathbf{I}=\mathbf{0} \quad \text { in } \Omega_{a} \\
\operatorname{grad} \mathbf{k}=\mathbf{0} \quad \text { in } \Omega_{a} \\
\mathbf{k}=\mathbf{0} \quad \text { on } \Gamma \\
\frac{1}{|\Omega|} \int_{\Omega_{a}} \mathbf{b d} \Omega=\mathbf{0}
\end{gathered}
$$

where $\mathbf{b}$ is a vector solution of the above boundary value problem that characterizes the fluctuation of pressure at the pore scale induced by the macroscopic gradient.

All the other parameters involved in this model are the same as the ones defined in Case A1.

In Case B1, the heat and water vapor transfer is described by two coupled equations of diffusion and convection, including a source term induced by the phase change. The air flow is described by the classical Darcy's law (48); however, the mass balance (47) presents also a source term due to the porosity variation induced by the phase change. Once again, based on the dimensionless number analysis, the source term $Q_{T}$ arising in the heat transfer equation is negligible in Case B2. In Case B3, the source terms $Q_{T}$ and $Q_{V}$ arising in the heat and vapor transfer equations are negligible. In both Cases B2 and B3, the source term in the mass balance (48) is also negligible.

\subsubsection{Case $\mathrm{C} 1$ (C2 and C3): Strong Convection + Dispersion + Source Terms}

In Case C1, diffusive effects on the heat and water vapor transfer are negligible at the first order of approximation compared to the convective ones. The transfers are thus governed by the air flow and described by the following set of equations (see equations (C23), (C24), (C34), and (C59) in the supporting information):

$$
\begin{gathered}
\operatorname{div}\left\langle\mathbf{v}_{a}^{(0)}\right\rangle=0 \\
\left\langle\mathbf{v}_{a}^{(0)}\right\rangle=-\mathbf{F}\left(\operatorname{grad} p_{a}^{(0)}, \text { microstructure, } \rho_{a}, \mu_{a}\right) \\
(\rho C)^{\mathrm{eff}} \frac{\partial T^{(0)}}{\partial t}+\rho_{a} C_{a}\left\langle\mathbf{v}_{a}^{(0)}\right\rangle \cdot \operatorname{grad} T^{(0)}=0 \\
\phi \frac{\partial \rho_{v}^{(0)}}{\partial t}+\left\langle\mathbf{v}_{a}^{(0)}\right\rangle \cdot \operatorname{grad} \rho_{v}^{(0)}=0
\end{gathered}
$$

where $\mathbf{F}$ is the flow law which is a nonlinear function of the macroscopic gradient of pressure (to take into account inertial effects) and depends on the microstructure and the physical properties of the fluid $\left(\rho_{a}, \mu_{a}\right)$. This function $\mathbf{F}$ can be determined by solving the boundary value problem over the REV (see equations (C15)-(C18) in the supporting information).

In Case C1, at the first order of approximation, at the macroscopic scale, the heat and water vapor transfer is described by two equations of convection, which do not include source terms induced by the phase change. The set of equations (56) - (59) is thus valid for Cases C1, C2, and C3.

Remark 1. In the presence of strong convection, the above macroscopic modeling shows that the macroscopic flow law $\mathbf{F}$ becomes strongly nonlinear. During the last decades, several theoretical and numerical works [see, for example, Mei and Auriault, 1991; Skjetne and Auriault, 1999; Lasseux et al., 2011, and references therein] 
have been performed in order to find a general expression of $\mathbf{F}$, whatever the porous media (isotropic or anisotropic). As recently shown by Lasseux et al. [2011], due to the nonlinearity, there is generally a strong coupling between the flow and the microstructure of the porous media. Consequently, the mean velocity is not necessarily collinear to the macroscopic pressure gradient as in the case of Darcy's law. As a consequence, in the case of orthotropic porous media, for example, the knowledge of this relationship within the principal axis, as it has been performed on 3-D images of snow by Zermatten et al. [2014], is not sufficient to describe the flow for every orientation of the pressure gradient with respect to the microstructure.

Remark 2. In porous media, it is well known that when the Péclet number at the pore scale is larger than 1 [Bear, 1972], the transfers through the porous media are usually driven by convection and dispersion. As shown in Auriault and Adler [1995], the interplay between diffusion and convection will appear by investigating the first corrector (i.e, the next order of the asymptotic expansion) of the above macroscopic modeling. In the present case, following Auriault and Adler [1995], Geindreau and Auriault [2001], and Auriault et al. [2009], it can be shown that at the second order of approximation, the macroscopic model for the heat and the water vapor transfer is written as follows (see equations (C53) and (C71) in the supporting information):

$$
\begin{gathered}
(\rho C)^{\mathrm{eff}} \frac{\partial\langle T\rangle}{\partial t}+\rho_{a} C_{a}\left\langle\mathbf{v}_{a}\right\rangle \cdot \operatorname{grad}\langle T\rangle-\operatorname{div}\left(\mathbf{k}^{\mathrm{disp}} \operatorname{grad}\langle T\rangle\right)=\mathrm{SSA}_{V} L_{s g} w_{n}^{(0)} \\
\phi \frac{\partial\left\langle\rho_{v}\right\rangle_{a}}{\partial t}+\left\langle\mathbf{v}_{a}\right\rangle \cdot \operatorname{grad}\left\langle\rho_{v}\right\rangle_{a}-\operatorname{div}\left(\mathbf{D}^{\operatorname{disp}} \operatorname{grad}\left\langle\rho_{v}\right\rangle_{a}\right)=-\mathrm{SSA}_{V} \rho_{i} w_{n}^{(0)}
\end{gathered}
$$

where $\langle T\rangle,\left\langle\rho_{v}\right\rangle_{a}$, and $\left\langle\mathbf{v}_{a}\right\rangle$ are the macroscopic temperature, water vapor density, and fluid velocity, respectively, and $\mathbf{k}^{\text {disp }}$ and $\mathbf{D}^{\text {disp }}$ are the effective thermal dispersion tensor and the effective dispersion tensor, respectively. The notations $\langle\cdot\rangle_{a}$ and $\langle\cdot\rangle$ represent the mean over the air phase and over the REV, respectively. These tensors depend on the local velocity field and are defined as follows (see equations (C51) and (C69) in the supporting information):

$$
\begin{gathered}
\mathbf{k}^{\text {disp }}=\frac{1}{|\Omega|}\left(\int_{\Omega_{a}} \kappa_{a}\left(\operatorname{grad} \mathbf{m}_{a}+\mathbf{I}\right)+\mathbf{v}_{a}^{(0)} \otimes \mathbf{m}_{a} \mathrm{~d} \Omega+\int_{\Omega_{i}} \kappa_{i}\left(\operatorname{grad} \mathbf{m}_{i}+\mathbf{I}\right) \mathrm{d} \Omega\right) \\
\mathbf{D}^{\text {disp }}=\frac{1}{|\Omega|} \int_{\Omega_{a}} D_{v}\left(\operatorname{grad} \mathbf{h}_{v}+\mathbf{I}\right)+\mathbf{v}_{a}^{(0)} \otimes \mathbf{h}_{v} \mathrm{~d} \Omega
\end{gathered}
$$

where $\mathbf{m}_{a}$ and $\mathbf{m}_{i}$ are periodic vectors solution of the following boundary value problem over the REV (see equations (C41)-(C45) in the supporting information):

$$
\begin{gathered}
-\beta\left\langle\mathbf{v}_{a}^{(0)}\right\rangle-\operatorname{div}\left(\kappa_{i}\left(\operatorname{grad} \mathbf{m}_{i}+\mathbf{I}\right)\right)=\mathbf{0} \quad \text { in } \Omega_{i} \\
-\gamma\left\langle\mathbf{v}_{a}^{(0)}\right\rangle+\rho_{a} C_{a} \mathbf{v}_{a}^{(0)} \cdot\left(\operatorname{grad} \mathbf{m}_{a}+\mathbf{I}\right)-\operatorname{div}\left(\kappa_{a}\left(\operatorname{grad} \mathbf{m}_{a}+\mathbf{I}\right)\right)=\mathbf{0} \quad \text { in } \Omega_{a} \\
\mathbf{m}_{i}=\mathbf{m}_{a} \text { on } \Gamma \\
\left(\kappa_{i}\left(\operatorname{grad} \mathbf{m}_{i}+\mathbf{I}\right)-\kappa_{a}\left(\operatorname{grad} \mathbf{m}_{a}+\mathbf{I}\right)\right) \cdot \mathbf{n}^{\Gamma}=\mathbf{0} \quad \text { on } \Gamma \\
\frac{1}{|\Omega|} \int_{\Omega}\left(\mathbf{m}_{a}+\mathbf{m}_{i}\right) \mathrm{d} \Omega=\mathbf{0}
\end{gathered}
$$

where $\beta=\left(\rho_{a} C_{a}\right) \times\left(\rho_{i} C_{i}\right) /(\rho C)^{\text {eff }}$ and $\gamma=\left(\rho_{a} C_{a}\right)^{2} /(\rho C)^{\text {eff }}$. Similarly, $\mathbf{h}_{v}$ is a periodic vector solution of the following boundary value problem over the REV (see equations (C63) - (C65) in the supporting information):

$$
\begin{gathered}
-\phi^{-1}\left\langle\mathbf{v}_{a}^{(0)}\right\rangle+\mathbf{v}_{a}^{(0)}+\mathbf{v}_{a}^{(0)} \cdot \operatorname{grad} \mathbf{h}_{v}-\operatorname{div}\left(D_{v}\left(\operatorname{grad} \mathbf{h}_{v}+\mathbf{I}\right)\right)=\mathbf{0} \quad \text { in } \Omega_{a} \\
D_{v}\left(\operatorname{grad} \mathbf{h}_{v}+\mathbf{I}\right) \cdot \mathbf{n}^{\Gamma}=\mathbf{0} \quad \text { on } \Gamma \\
\frac{1}{|\Omega|} \int_{\Omega_{a}} \mathbf{h}_{v} \mathrm{~d} \Omega=\mathbf{0}
\end{gathered}
$$




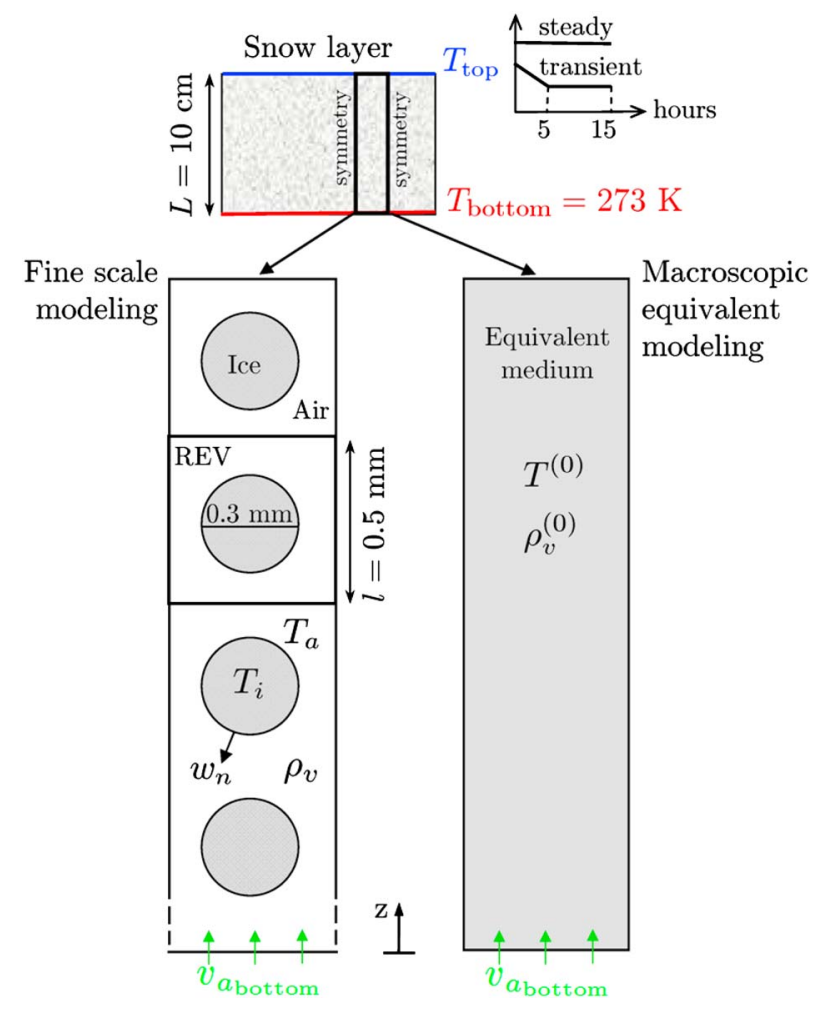

Figure 4. Illustration of the 2-D geometry for the fine-scale modeling and the macroscopic equivalent modeling.

These two boundary value problems clearly underline the strong coupling between the diffusion and the convection phenomena at the REV scale and consequently at the macroscopic scale through the dispersion tensors.

\section{Two-Dimensional Numerical Illustration}

\subsection{Problem Definition}

In order to illustrate and evaluate the obtained macroscopic modeling, we propose to compare the numerical results for the heat and water vapor transfer through a snow layer obtained in the case of a fine-scale modeling (i.e., by taking into account all the heterogeneities) and in the case of the macroscopic equivalent modeling. We focus on Case B1, i.e., when a moderate convection is considered.

For that purpose, numerical simulations have been preformed on the same 2-D vertical snow layer of $10 \mathrm{~cm}$ length and $0.5 \mathrm{~mm}$ width (Figure 4) using the finite element code ComsolMultiphysics. The separation of scales $\varepsilon$ remains equal to $5 \times 10^{-3}$, as in the theoretical developments. At the base of the snow layer, the temperature $T_{\text {bottom }}$ is equal to $273 \mathrm{~K}$; the vapor density $\rho_{v s}\left(T_{\text {bottom }}\right)$ is equal to $5.113 \times 10^{-3} \mathrm{~kg} \mathrm{~m}^{-3}$, which is supposed to be equal to the saturation vapor density using equation (12); and the intensity of the air flow through the snow layer given by $v_{a_{\text {bottom }}}$ are imposed. At the top of the snow layer, the air pressure $p_{a_{\text {top }}}$ is arbitrarily fixed to $1 \mathrm{~Pa}$ and the temperature $T_{\text {top }}$ is adjusted in order to impose a given macroscopic temperature gradient defined as $\left(\left(T_{\text {top }}-T_{\text {bottom }}\right) / L\right.$ ) through the snow layer. The vapor density is not imposed at the outlet as in Albert and McGilvary [1992]. Symmetry conditions are imposed on the lateral sides of the snow layer. The above boundary conditions are similar to the ones defined in the study of Albert and McGilvary [1992]. In what follows, curvature effects are ignored, i.e., $d_{0} K=0$ (negligible compared to the temperature gradient effects), and the interface kinetic coefficient $\beta$ is taken to be constant and equal to $5.5 \times 10^{5} \mathrm{~s} \mathrm{~m}^{-1}$, as in the study of Kaempfer and Plapp [2009]. Moreover, for the sake of simplicity, the simulations do not reflect the evolution of the microstructure (air-ice interface motion), i.e., the geometry is not updated with time.

In the case of the fine-scale modeling, the snow layer microstructure consists in 200 periodic cells of $0.5 \times 0.5$ $\mathrm{mm}^{2}$; each periodic cell (REV) is composed by an ice grain of diameter $0.3 \mathrm{~mm}$ surrounded by air. The porosity is thus equal to 0.71 , which corresponds to a snow density of $266 \mathrm{~kg} \mathrm{~m}^{-3}$. The heat and the vapor transfer 


Table 2. Macroscopic Properties Computed Over the REV of the
considered 2-D Geometry, Using $\kappa_{a}=0.024 \mathrm{~W} \mathrm{~m}^{-1} \mathrm{~K}^{-1}, \kappa_{i}=2.3 \mathrm{~W} \mathrm{~m}^{-1}$
$\mathrm{~K}^{-1}$, and $D_{V}=2.036 \times 10^{-5} \mathrm{~m}^{2} \mathrm{~s}^{-1}$
\begin{tabular}{lc} 
Macroscopic Properties & Values \\
\hline Porosity, $\phi$ & 0.71 \\
Surface area, SSA & $3770 \mathrm{~m}^{-1}$ \\
Intrinsic permeability, $K^{\text {eff }}$ & $2.71 \times 10^{-9} \mathrm{~m}^{-2}$ \\
Effective thermal capacity, $(\rho C)^{\text {eff }}$ & $5.3 \times 10^{6} \mathrm{~J} \mathrm{~m}^{2} \mathrm{~K}^{-1}$ \\
Effective thermal conductivity, $k^{\text {eff }}$ & $0.04243 \mathrm{~W} \mathrm{~m}^{-1} \mathrm{~K}^{-1}$ \\
Effective diffusion, $D^{\text {eff }}$ & $1.156 \times 10^{-5} \mathrm{~m}^{2} \mathrm{~s}^{-1}$ \\
\hline
\end{tabular}

within this layer is described by the set of equations (1)-(12), where $p_{a}, v_{a}, T_{i}, T_{a}$, and $\rho_{v}$ are the unknowns. This set of equations has been numerically solved using the material parameter values presented in Table 1.

In the case of the macroscopic equivalent modeling, the snow layer is seen as a continuous equivalent medium. We simulated Case B1: the heat and the mass transfer is described by the set of equations (47) - (50), (34), and (35), where $p_{a}^{(0)}, v_{a}^{(0)}, T^{(0)}$, and $\rho_{v}^{(0)}$ are the macroscopic unknowns. This macroscopic description requires to compute some parameters or effective properties over the REV. The porosity $\phi$ and the specific surface area SSA $\mathrm{S}_{V}$ involved in the macroscopic modeling can be easily deduced from the geometry of the REV. The intrinsic permeability tensor $\mathbf{K}^{\text {eff }}$, effective heat capacity $(\rho C)^{\text {eff }}$, effective thermal conductivity tensor $\mathbf{k}^{\text {eff }}$, and effective vapor diffusion tensor $\mathbf{D}^{\text {eff }}$ were computed using relations (51), (36), (37), and (38), respectively. For that purpose, the boundary value problems (52) - (55), (39) - (43), and (44) - (46) have been solved numerically over the REV. Due to the symmetry of the REV, tensors $\mathbf{K}^{\text {eff }}$, $\mathbf{D}^{\text {eff }}$, and $\mathbf{k}^{\text {eff }}$ are isotropic and can be written as $\mathbf{K}^{\text {eff }}=K^{\text {eff }} \mathbf{I}$, $\mathbf{D}^{\text {eff }}=D^{\text {eff }} \mathbf{I}$, and $\mathbf{k}^{\text {eff }}=k^{\text {eff }} \mathbf{I}$, where $\mathbf{I}$ is the identity tensor. The parameters and the effective properties deduced from the REV scale are summarized in Table 2.

\subsection{Results}

\subsubsection{Illustration of the Physics Involved}

First, Figures 5 and 6 illustrate the physical phenomena involved at the microscopic and macroscopic scales. Figure 5 shows the velocity field, temperature field, and vapor density field, with the corresponding fluxes (black arrows), as well as the vapor density flux at the interface. These variables are computed within two cells of the snow layer using the fine-scale modeling when $T_{\text {top }}=248 \mathrm{~K}$, leading to a temperature gradient of $100 \mathrm{~K} \mathrm{~m}^{-1}$, in steady state. For the temperature field and the vapor density field, we use systematically a color map defined as the average temperature over the cell $\pm 0.15 \mathrm{~K}$ and the average vapor density $\pm 0.03 \times 10^{-3} \mathrm{~kg} \mathrm{~m}^{-3}$, respectively. The vapor fluxes at the interface are represented by red arrows that are proportional to the flux intensity. In this way, the intensity of the temperature and vapor density gradients and of the interfacial vapor flux of each caption can be easily compared.

When a very slow flow is simulated $\left(v_{a_{\text {bottom }}}=10^{-8} \mathrm{~m} \mathrm{~s}^{-1}\right.$, i.e., $\left.[R e]=\left[P e^{\top}\right]=\left[P e^{\rho}\right]=\mathcal{O}\left(\varepsilon^{3}\right)\right)$, the intensity of gradients of temperature and vapor density are similar between the cell at the top and bottom of the layer; only the values are different. In detail, the intensity of both gradients is slightly higher in the cell at the base of the layer than in the one at the top. This small difference is due to the nonlinear relationship (exponential) between temperature and saturation vapor density. When a very slow flow is considered, the heat transfer is clearly higher in the ice phase than in the air phase. Along the interface, the vapor fluxes reflect the ice sublimation on the upper part of the grain and the water vapor deposition on the lower part. Locally, the fluxes of vapor and heat are influenced by these phase changes (source terms), in addition to the macroscopic temperature gradient imposed.

When we considered the simulations with a high air flow velocity $\left(v_{a_{\text {bottom }}}=10^{-3} \mathrm{~m} \mathrm{~s}^{-1}\right.$, i.e., $[R e]=\left[P e^{\top}\right]=$ $\left[\mathrm{Pe}^{\rho}\right] \leq \mathcal{O}\left(\varepsilon^{2}\right)$ ), the intensity of the gradients of temperature and vapor density are different between the top and base of the snow layer. For the cell located at the bottom, the temperature and vapor density are quasi-homogeneous. Inversely, the cell located at the top exhibits large gradients. This configuration is due to the input air flow at the bottom of the layer that transfers the heat and vapor toward the top; the gradients are thus concentrated in this area. The heat and vapor fluxes are identical to those of the air flow, i.e., the transfer is driven by the air flow, in addition to the macroscopic temperature gradient imposed, and the effect of phase changes is not observable anymore. At the base of the snow layer, because the temperature gradient is low, 


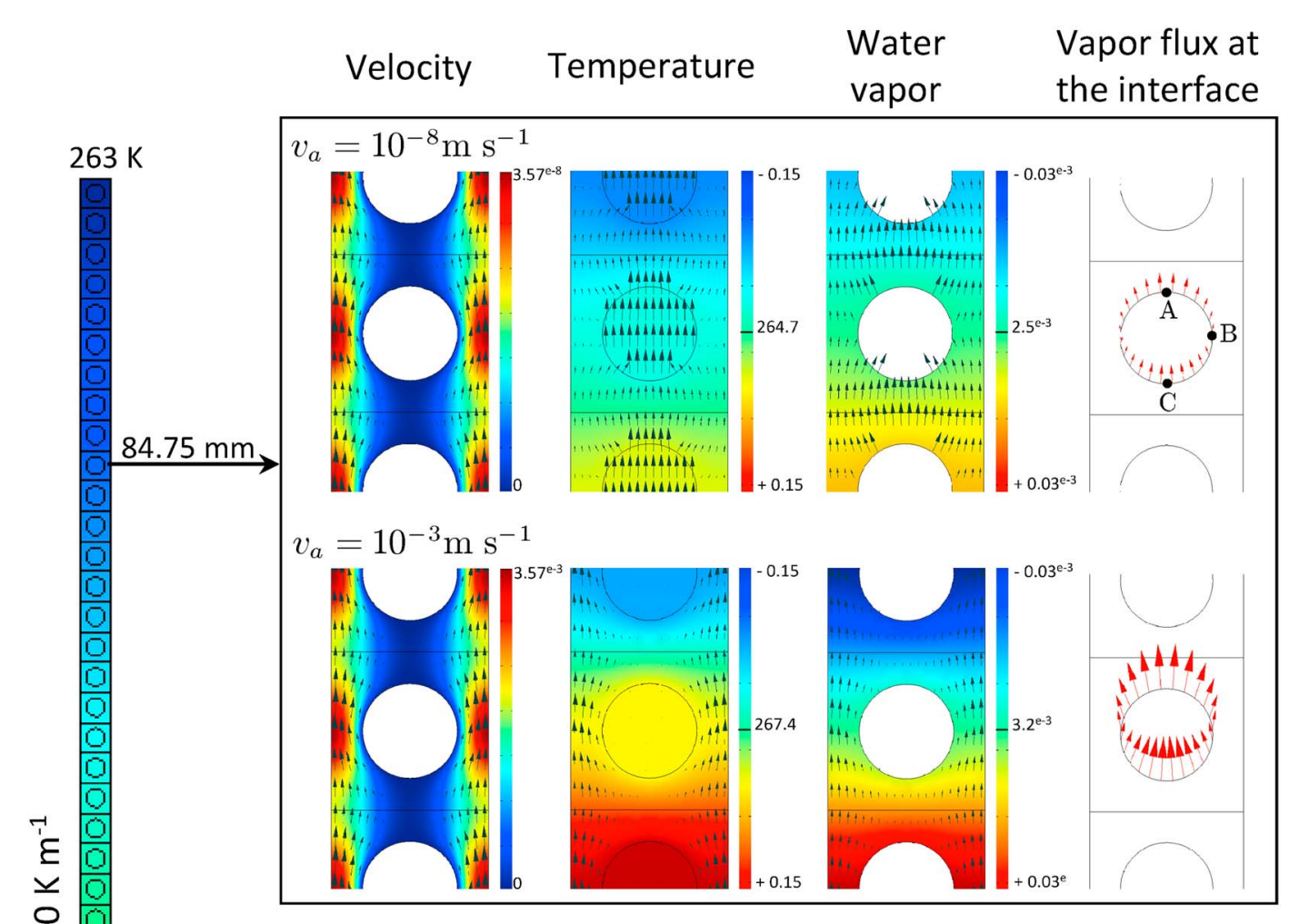




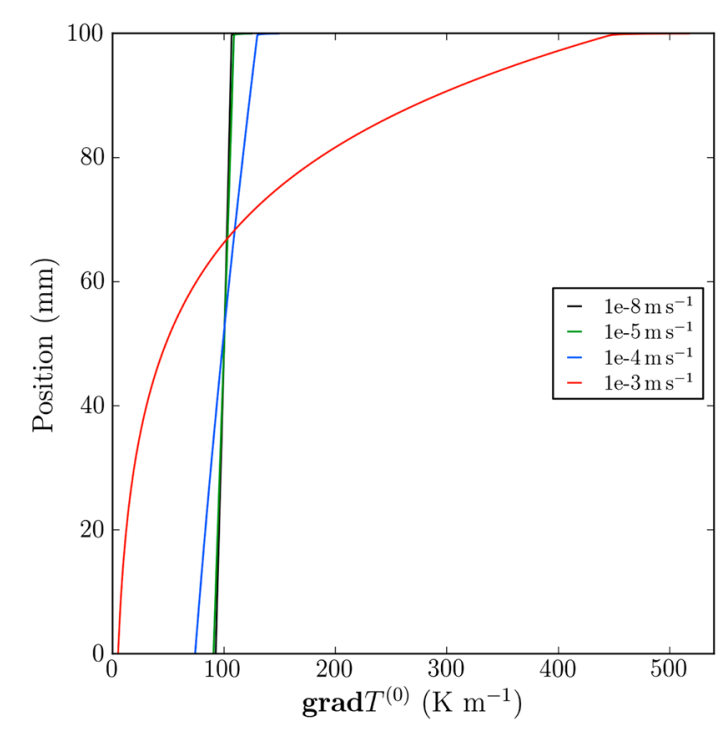

Figure 6. Vertical profile of the local temperature gradient depending on the input air velocity.

the vapor flux at the interface due to phase change is also very low. Inversely, the phase change is very active at the top of the layer, due to large temperature gradients.

The above comments show the strong influence of the air flow on the temperature field and, as it is coupled, on the vapor density field at the microscopic scale. This effect is also observed the macroscale in Figure 6 , which shows the vertical profile of the intensity of local temperature gradients induced by a macroscopic temperature gradient of $100 \mathrm{~K} \mathrm{~m}^{-1}$ and under three different values of air flow velocity $v_{a_{\mathrm{bottom}}}$. As expected, when low flow velocities are considered, the snow layer is submitted to a quasi-constant vertical temperature gradient very close to the macroscopic one imposed; but when a strong convection occurs, the snow locally undergoes temperature gradients that increase along the flow direction from the base to the top of the layer. In the case of Figure 6, the local gradients range from about 0 to $500 \mathrm{~K} \mathrm{~m}^{-1}$ for a macroscopic temperature gradient of $100 \mathrm{~K} \mathrm{~m}^{-1}$.

\subsubsection{Comparison of the Microscopic and Macroscopic Models}

The following results, presented in Figures 7-10, aim at comparing the vertical profiles of the temperature and vapor density through the snow layer obtained in the case of a fine-scale modeling (marks) and in the case of the corresponding macroscopic equivalent modeling (solid line). In the case of the fine-scale modeling, the average values of each microscopic variable over the cell have been reported, expressed as follows:

$$
\langle T\rangle=\frac{1}{\Omega}\left(\int_{\Omega_{i}} T_{i} \mathrm{~d} \Omega+\int_{\Omega_{a}} T_{a} \mathrm{~d} \Omega\right), \quad\left\langle\rho_{v}\right\rangle=\frac{1}{\Omega_{a}} \int_{\Omega_{a}} \rho_{v} \mathrm{~d} \Omega
$$

Figures 7-9 show the obtained results in steady state, when $T_{\text {bottom }}=273 \mathrm{~K}$ and $T_{\text {top }}=268,263$, and $248 \mathrm{~K}$, which leads to a temperature gradient of 50,100 , and $250 \mathrm{~K} \mathrm{~m}^{-1}$, respectively. For each figure, the velocity of the input air flow at the base of the snow layer $v_{a_{\text {bottom }}}$ is equal to $10^{-8}, 10^{-5}, 10^{-4}$, and $10^{-3} \mathrm{~m} \mathrm{~s}^{-1}$ successively. An air flow above $10^{-4} \mathrm{~m} \mathrm{~s}^{-1}$, i.e., $[R e]=\left[P e^{\top}\right]=\left[P e^{\rho}\right] \geq \mathcal{O}(\varepsilon)$, significantly modifies the temperature and vapor profile, as expected by the dimensionless numbers analysis. Below this value, the profiles are the same as the ones presented in Calonne et al. [2014a] with no convection. Overall, profiles deduced from the fine-scale modeling and the macroscopic equivalent modeling are close. The maximum relative error is reached in the upper part of the snow layer for $v_{a_{\text {bottom }}}=10^{-3} \mathrm{~m} \mathrm{~s}^{-1}$ and a temperature gradient of $250 \mathrm{~K} \mathrm{~m}^{-1}$ and is equal to $-0.39 \%$ for the temperature and $-9.4 \%$ for the vapor density. These errors are in absolute value larger than and of opposite sign to the ones obtained when no convection is considered [Calonne et al., 2014a] or similar in the present case when $v_{a_{\text {bottom }}}=10^{-8} \mathrm{~m} \mathrm{~s}^{-1}$.

Figures 10 and 11 show the temperature and vapor density profiles for a temperature gradient of $100 \mathrm{~K} \mathrm{~m}^{-1}$ and with $v_{a_{\text {bottom }}}=10^{-3}$ and $10^{-8} \mathrm{~m} \mathrm{~s}^{-1}$, respectively, when the heat and vapor transfer is solved in transient 

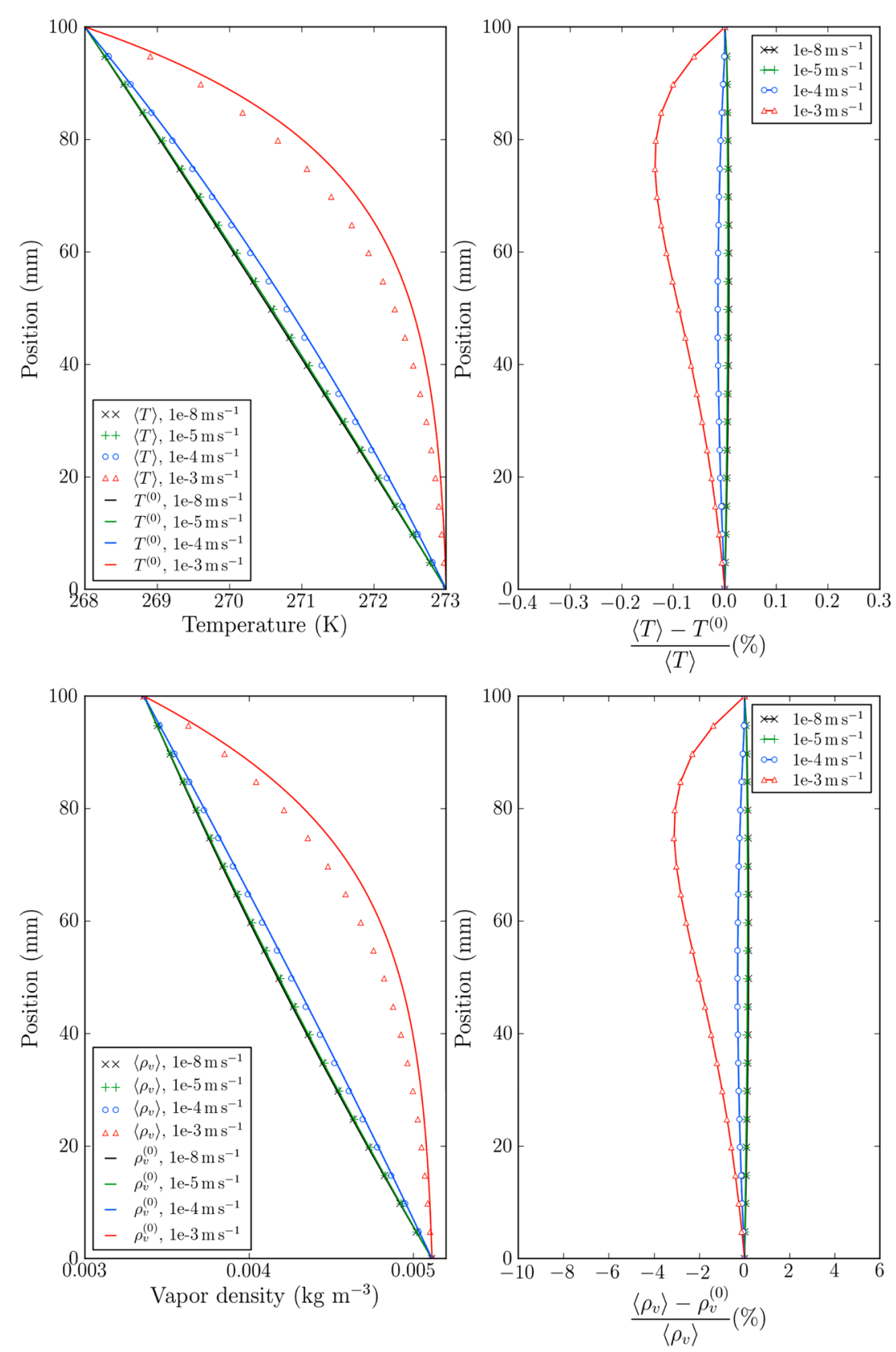

Figure 7. Comparison between fine-scale (marks) and macroscopic equivalent (solid line) modeling in steady state and for a temperature gradient of $50 \mathrm{~K} \mathrm{~m}^{-1}$ : vertical profile of temperature and vapor density and relative errors for an input air velocity $v_{a_{\text {bottom }}}$ of $10^{-8}, 10^{-5}, 10^{-4}$, and $10^{-3} \mathrm{~m} \mathrm{~s}^{-1}$.

state and the air flow is solved in steady state. The snow layer is initially at a temperature of $273 \mathrm{~K}$; then $T_{\text {top }}$ decreases to reach $263 \mathrm{~K}$ in $5 \mathrm{~h}$ and remains constant until $15 \mathrm{~h}$. The value of $T_{\text {bottom }}$ is constant and equal to $273 \mathrm{~K}$. The profiles at five different times are shown in both figures. Once again, we can observe a good agreement between the numerical results of the fine-scale and the equivalent macroscopic modelings. Under such conditions, the maximum relative error is obtained at $t=6 \mathrm{~h}$ and reaches $-0.14 \%$ for the temperature profile and $-3.35 \%$ for the vapor density profile.

Finally, Figure 12 presents the evolution of the normal growth velocity of the ice grain in two cells positioned at the upper and lower parts of the snow layer deduced from the fine-scale modeling $\left(w_{n}\right.$ at the top (point A), midheight (point B), and base (point C) of the ice grain as well as the average value $\left\langle w_{n}\right\rangle$ over the REV, Figure 5) and from the macroscopic equivalent modeling $\left(w_{n}^{(0)}\right)$ for a macroscopic temperature gradient within 

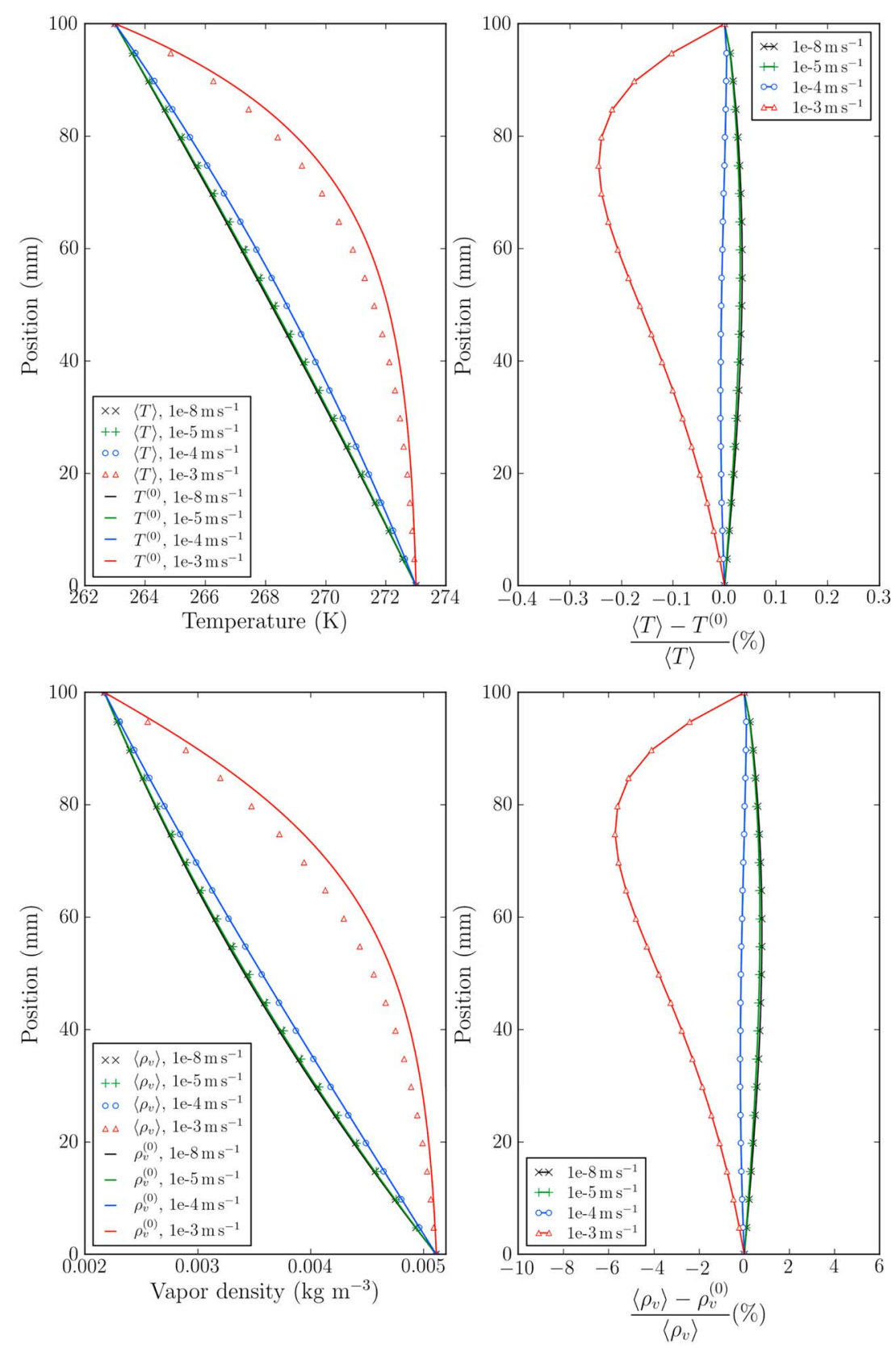

Figure 8. Comparison between fine-scale (marks) and macroscopic equivalent (solid line) modeling in steady state and for a temperature gradient of $100 \mathrm{~K} \mathrm{~m}^{-1}$ : vertical profile of temperature and vapor density and relative errors for an input air velocity $v_{a_{\text {bottom }}}$ of $10^{-8}, 10^{-5}, 10^{-4}$, and $10^{-3} \mathrm{~m} \mathrm{~s}^{-1}$.

the range 0 to $250 \mathrm{~K} \mathrm{~m}^{-1}$ and for $v_{a_{\text {bottom }}}$ equal to $10^{-8}$ and $10^{-3} \mathrm{~m} \mathrm{~s}^{-1}$. The values of $w_{n}$ at the top of the grain are negative and presented in terms of absolute values in the figure. As expected, the normal growth velocity increases with increasing temperature gradient. For the upper cell, the local temperature gradients can be strongly enhanced compared to the macroscopic gradient imposed, as shown by Figure 6 . In spite of this, the average value $\left\langle w_{n}\right\rangle$ of this cell does not exceed $2 \times 10^{-11} \mathrm{~m} \mathrm{~s}^{-1}\left([W]=[H] \simeq \mathcal{O}\left(\varepsilon^{2}\right)\right)$. For both cells, $w_{n}$ is negative (sublimation) and positive (deposition) at the top and at the base of the ice grain, respectively, and ranges typically from $10^{-11}$ to $10^{-9} \mathrm{~m} \mathrm{~s}^{-1}$ (in absolute value), whereas at the midheight of the grain $w_{n}$ ranges from $10^{-15}$ to $10^{-11} \mathrm{~m} \mathrm{~s}^{-1}$. The averaged growth velocity over the particle interface is low but positive, meaning that the deposition predominates slightly over the sublimation. This is due to the nonlinear relationship between the temperature and the saturation vapor density. The evolution of the "macroscopic equivalent normal velocity" $w_{n}^{(0)}$ is consistent with $\left\langle w_{n}\right\rangle$. 

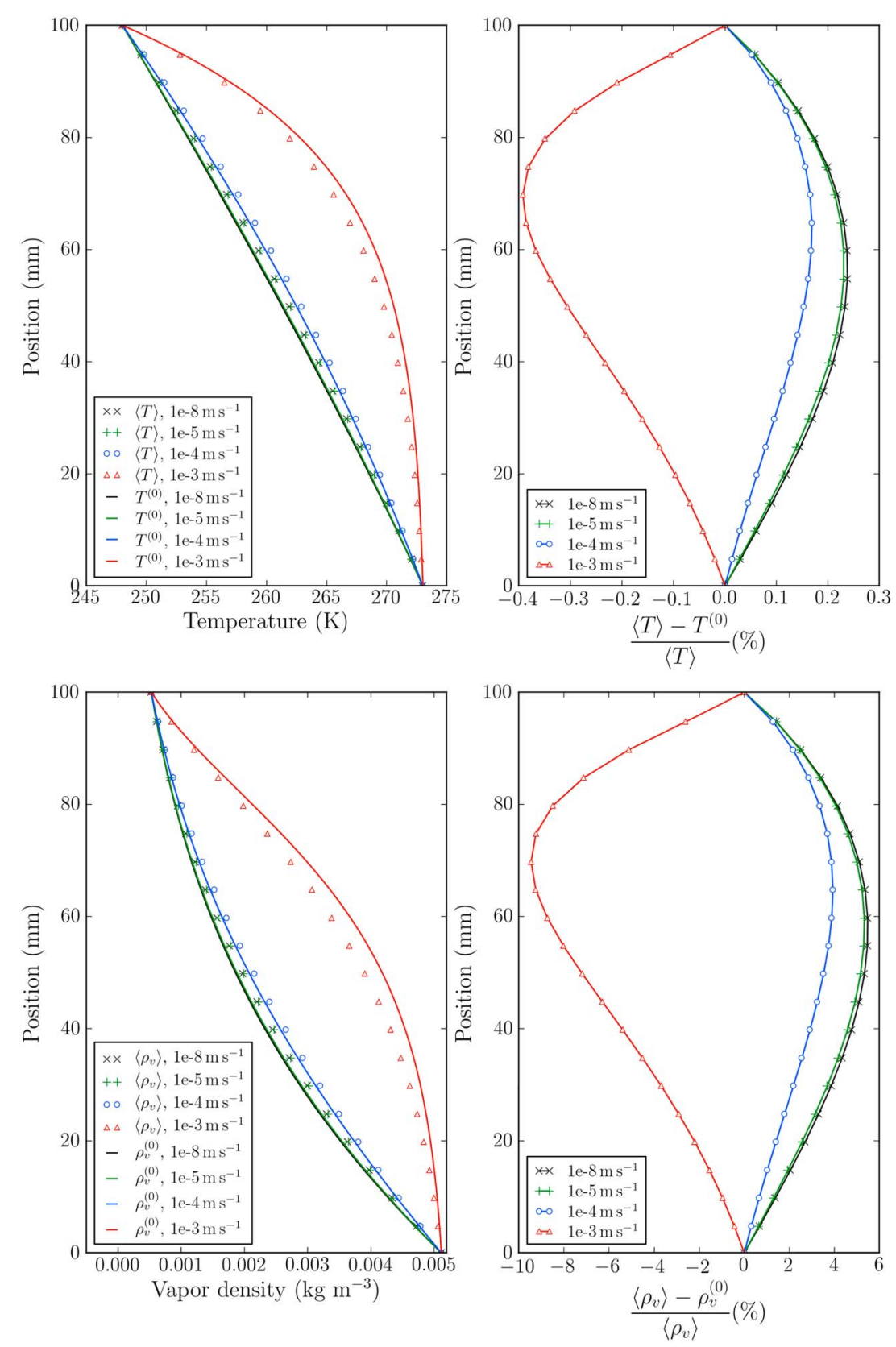

Figure 9. Comparison between fine-scale (marks) and macroscopic equivalent (solid line) modeling in steady state and for a temperature gradient of $250 \mathrm{~K} \mathrm{~m}^{-1}$ : vertical profile of temperature and vapor density and relative errors for an input air velocity $v_{a_{\text {bottom }}}$ of $10^{-8}, 10^{-5}, 10^{-4}$, and $10^{-3} \mathrm{~m} \mathrm{~s}^{-1}$.

\section{Discussion and Conclusion}

We derived the macroscopic equivalent modeling of the heat and water vapor transfer through snow from its description at the pore scale using the homogenization of multiple-scale expansions [Bensoussan et al., 1978; Sanchez-Palencia, 1980; Auriault, 1991; Auriault et al., 2009]. Complementing the study of Calonne et al. [2014a], an air flow is considered in the definition of the physics at the pore scale. The formulation of the macroscopic equivalent modeling is driven by the order of magnitude of the dimensionless numbers that characterize the physics at the pore scale and that thus constitute the domain of validity of the modeling. The formulation includes the exact expression of the effective properties (effective thermal conductivity, effective vapor diffusion coefficient, and intrinsic permeability) and of the source terms of heat and vapor arising from the phase change at the pore scale. Such definitions can be used to compute macroscopic properties from 

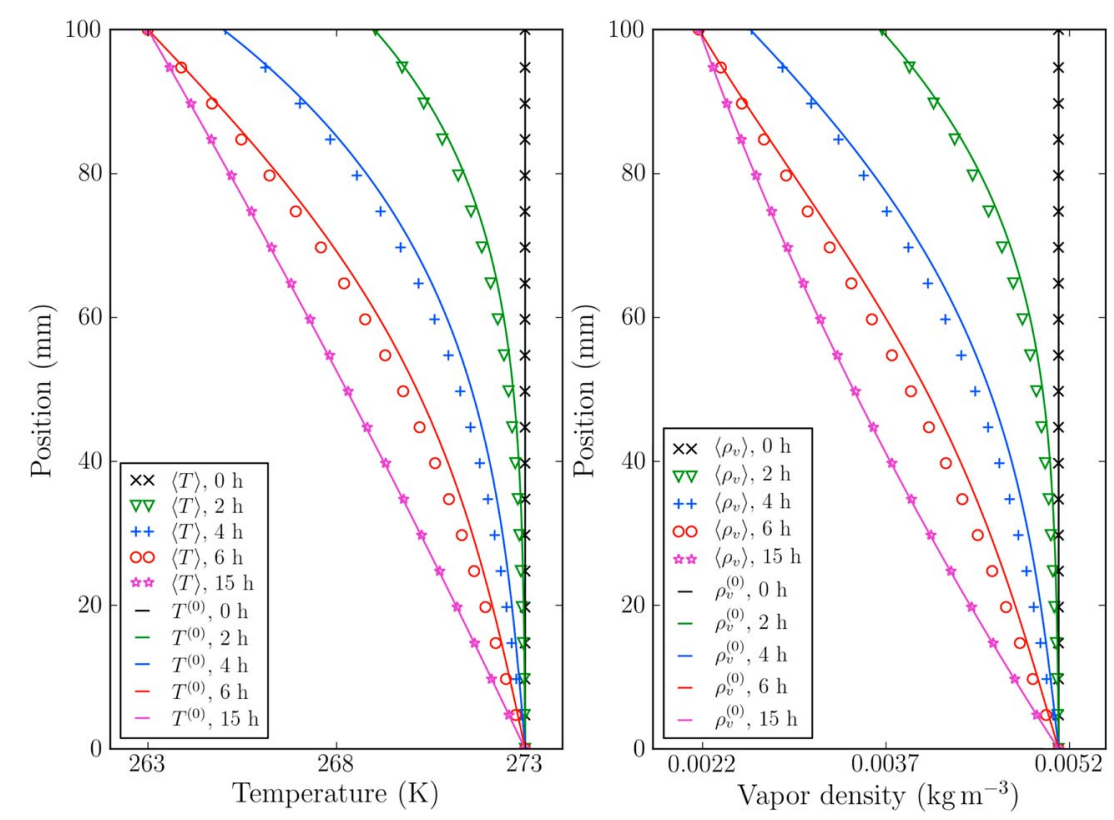

Figure 10. Comparison between fine-scale (marks) and macroscopic equivalent (solid lines) modeling in the case of transient analysis for an input air velocity $v_{a_{\text {bottom }}}$ of $10^{-8} \mathrm{~m} \mathrm{~s}^{-1}$ : vertical profile of temperature and water vapor density within the snow layer at five different times.

the 3-D description of the snow microstructure, e.g., obtained by X-ray tomography [Calonne et al., 2011, 2012, 2014a, 2014b].

The different estimations of the dimensionless numbers lead to three main cases of macroscopic modeling:

1. Case $A 1$ : when the air flow velocity $v_{a_{c}} \leq 10^{-5} \mathrm{~m} \mathrm{~s}^{-1}$, i.e., $\left[F_{i}^{\top}\right]=\left[F_{a}^{\top}\right]=\left[F_{a}^{\rho}\right]=\mathcal{O}\left(\varepsilon^{2}\right)$ and $[R e]=\left[P e^{\top}\right]=\left[P e^{\rho}\right] \leq$ $\mathcal{O}\left(\varepsilon^{2}\right)$, the convection is negligible at the macroscopic scale. The heat and vapor transfer at the scale of a snow layer is described by two coupled diffusion/conduction equations including source terms $\left(Q_{T}, Q_{v}\right)$ and corresponds to the one presented by Calonne et al. [2014a].
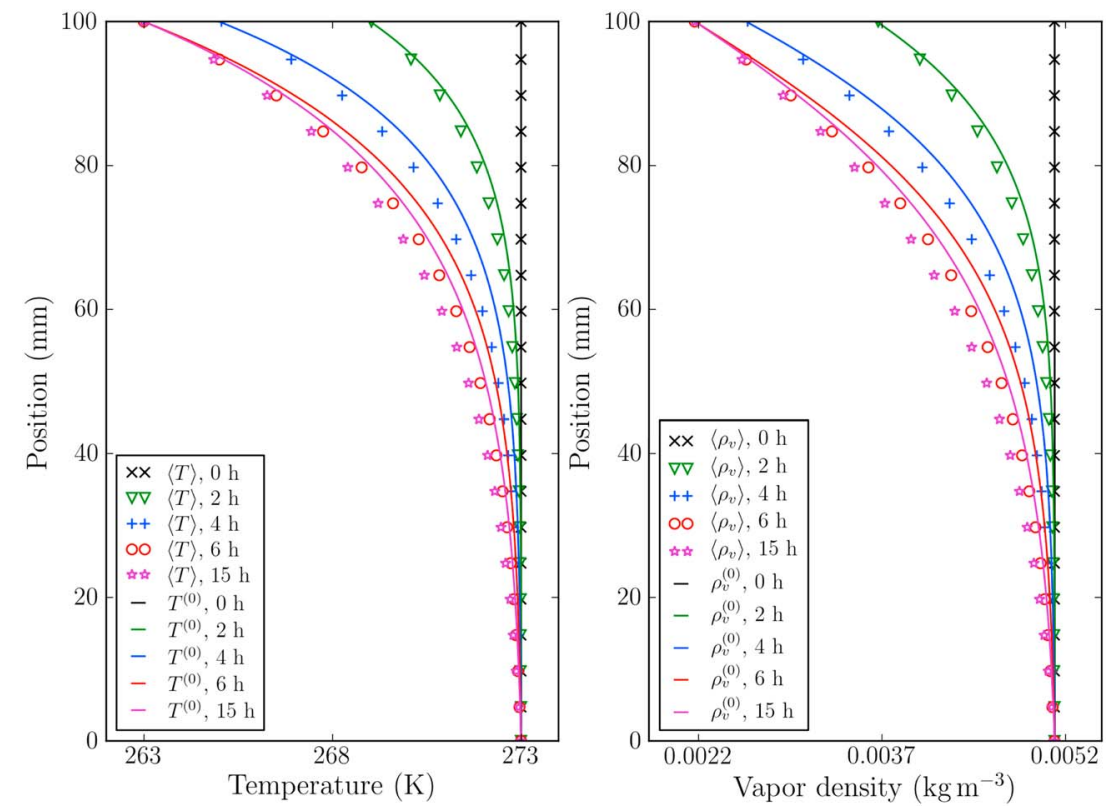

Figure 11. Comparison between fine-scale (marks) and macroscopic equivalent (solid lines) modeling in the case of transient analysis for an input air velocity $v_{a_{\text {bottom }}}$ of $10^{-3} \mathrm{~m} \mathrm{~s}^{-1}$ : vertical profile of temperature and water vapor density within the snow layer at five different times. 


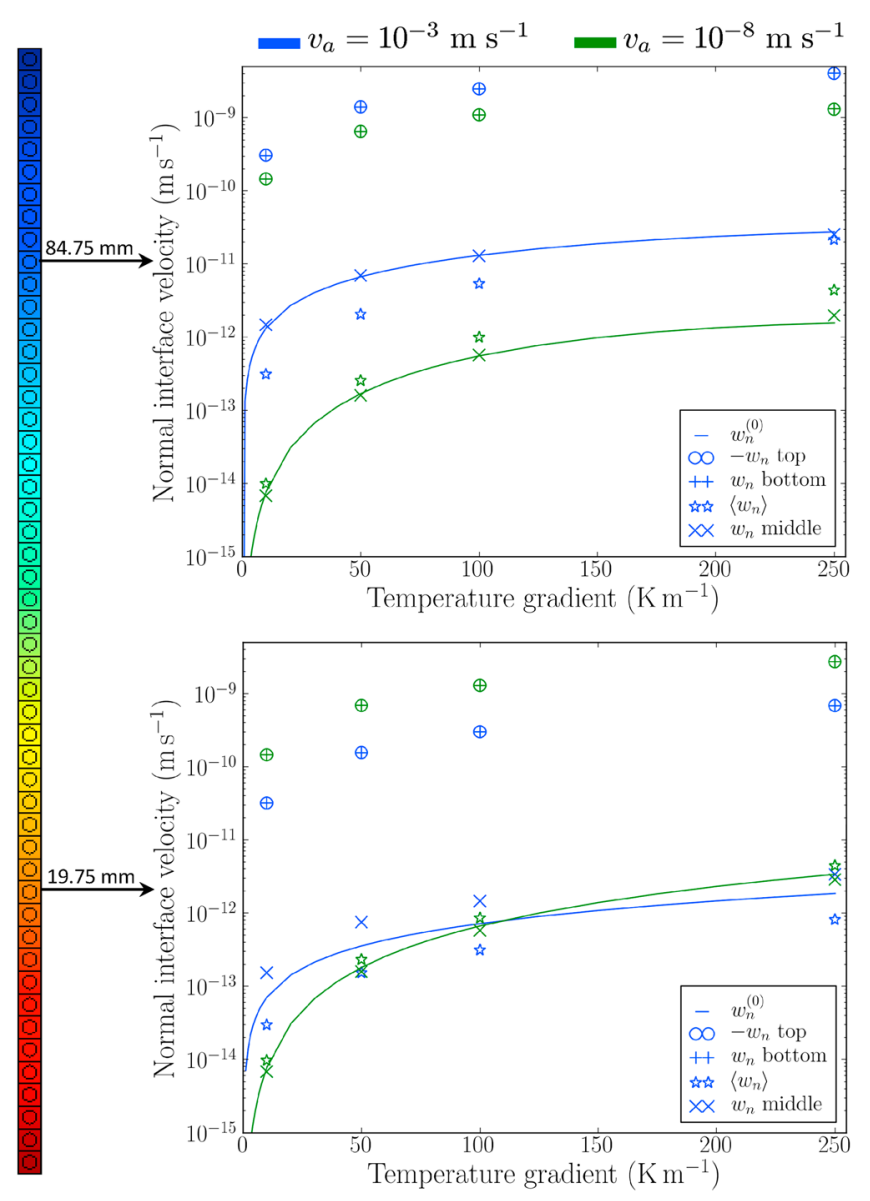

Figure 12. Evolution of the normal growth velocity in two cells positioned at the upper and lower parts of the 2-D snow layer deduced from the fine-scale modeling ( $w_{n}$ at the top, bottom, and middle of the ice grain as well as the average value $\left\langle w_{n}\right\rangle$ over the REV) and from the macroscopic equivalent modeling $\left(w_{n}^{(0)}\right)$. Results for a temperature gradient within the range $0-250 \mathrm{~K} \mathrm{~m}^{-1}$ and for an input air velocity $v_{a_{\text {bottom }}}$ of $10^{-8}$ and $10^{-3} \mathrm{~m} \mathrm{~s}^{-1}$.

2. Case B1: when $10^{-5} \mathrm{~m} \mathrm{~s}^{-1} \leq v_{a_{c}} \leq 10^{-3} \mathrm{~m} \mathrm{~s}^{-1}$, i.e., $\left[F_{i}^{\top}\right]=\left[F_{a}^{\top}\right]=\left[F_{a}^{\rho}\right]=\mathcal{O}\left(\varepsilon^{2}\right)$ and $[R e]=\left[P e^{\top}\right]=\left[P e^{\rho}\right]=\mathcal{O}(\varepsilon)$, the diffusion and convection are of the same order of magnitude at the macroscopic scale. The convection corresponds to a moderate flow that is described by Darcy's law. The heat and vapor transfer at the scale of a snow layer is described by two coupled convection-diffusion/conduction equations including source terms $\left(Q_{T}, Q_{v}\right)$.

3. Case $\mathrm{C}$ : when $10^{-3} \mathrm{~m} \mathrm{~s}^{-1} \leq v_{a_{c}} \leq 10^{-1} \mathrm{~m} \mathrm{~s}^{-1}$, i.e., $\left[F_{i}^{\top}\right]=\left[F_{a}^{\top}\right]=\left[F_{a}^{\rho}\right]=\mathcal{O}(\varepsilon)$ and $[R e]=\left[P e^{\top}\right]=\left[P e^{\rho}\right]=\mathcal{O}(1)$, the macroscopic diffusion is negligible at the first order of approximation. The convection corresponds to a strong flow that is not described anymore by Darcy's law but by a nonlinear flow to take into account inertial effects. The heat and vapor transfer is described at the macroscopic scale, at the second order of approximation, by two coupled convection-dispersion equations including source terms.

The subcases A2, A3, or B2, B3, or C2, C3, are particular cases of the three main cases described above: they correspond to simplifications of the source terms when the interface growth velocity is moderate to low, typically for $w_{n_{c}} \leq 10^{-11} \mathrm{~m} \mathrm{~s}^{-1}$, i.e., $[H] \leq \mathcal{O}\left(\varepsilon^{3}\right)$ and $[W] \leq \mathcal{O}\left(\varepsilon^{2}\right)$.

The modeling B1 — when the diffusion and convection are of the same order of magnitude at the macroscopic scale - is similar to the model presented by Albert and McGilvary [1992]. In their model, the expression of the source terms involved a mass transfer coefficient $h_{m}$. According to our developments, $h_{m}$ is perfectly defined and only depends on the macroscopic temperature as follows:

$$
h_{m}=\frac{\rho_{i}}{\beta \rho_{v s}^{(0)}\left(T^{(0)}\right)}
$$




\section{Acknowledgments}

We thank three anonymous reviewers for their comments that improved the quality of the manuscript. Funding by Météo-France and DigitalSnow (ANR-11-BS02-009) is acknowledged. CNRM-GAME/CEN is part of the Labex OSUG@2020 (Investissements d'Avenir-grant agreement ANR-10-LABX-56). 3SR lab is part of the LabEx Tec 21 (Investissements d'Avenir-grant agreement ANR-11-LABX-0030). No data were used in producing this manuscript.
This theoretical result contradicts the measurements of Neumann et al. [2009], which indicate that $h_{m}$ mainly depends on the air velocity through the snow sample, i.e., of the pore Reynolds number. However, the mean temperature was not homogeneous within their snow volume. So, it seems not obvious to define a mean mass transfer coefficient from their measurement, except by performing an inverse analysis.

Finally, the 2-D simulations of the physics at the pore scale and of the macroscopic modeling B1 illustrate the influence of the air flow on the heat and vapor transfer. If an air flow greater than about $10^{-4} \mathrm{~m} \mathrm{~s}^{-1}$ is applied, i.e., $[R e]=\left[P e^{\top}\right]=\left[P e^{\rho}\right] \geq \mathcal{O}(\varepsilon)$, the temperature field is significantly perturbed and the intensity of the temperature gradient varies widely locally. Despite the large local temperature gradients that can appear, the macroscopic equivalent normal velocity does not exceed $2 \times 10^{-11} \mathrm{~m} \mathrm{~s}^{-1}$. This result tends to show that Case 0 , i.e., when $w_{n_{c}} \approx 10^{-10} \mathrm{~m} \mathrm{~s}^{-1},[H]=\mathcal{O}\left(\varepsilon^{2}\right)$ and $[W]=\mathcal{O}(\varepsilon)$, where the transfers are driven by the interfacial phenomena, is unlikely to occur in natural snowpacks. Overall, the comparative study shows the precision and the robustness of the obtained macroscopic equivalent modeling B1.

\section{References}

Akitaya, E. (1974), Studies of depth hoar, Low. Temp. Sci. Ser. A, 26, 1-67.

Albert, M. R. (1993), Some numerical experiments on firn ventilation with heat transfer, Ann. Glaciol., 18, 161-165.

Albert, M. R., and W. McGilvary (1992), Thermal effects due to air flow and vapor transport in dry snow, J. Glaciol., 38(129), $273-281$.

Albert, M. R., and E. F. Shultz (2002), Snow and firn properties and air-snow transport processes at Summit, Greenland, Atmos. Environ., $36,2789-2797$

Albert, M. R., C. Shuman, Z. Courville, R. Bauer, M. Fahnestock, and T. Scambos (2004), Extreme firn metamorphism: Impact of decades of vapor transport on near-surface firn at a low-accumulation glazed site on the east antarctic plateau, Ann. Glaciol., 39(1), 73-78, doi:10.3189/172756404781814041.

Auriault, J. (2011), Heterogeneous periodic and random media. Are the equivalent macroscopic descriptions similar?, Int. J. Eng. Sci., 49, 806-808.

Auriault, J.-L. (1991), Heterogeneous medium. Is an equivalent description possible?, Int. J. Engng. Sci., 29, 785-795.

Auriault, J.-L., and P. Adler (1995), Taylor dispersion in porous media: Analysis by multiple scale expansions, Adv. Water Resour., 18(4), $217-226$.

Auriault, J.-L., C. Boutin, and C. Geindreau (2009), Homogenization of Coupled Phenomena in Heterogenous Media, Wiley-ISTE, London Bear, J. (1972), Dynamics of Fluids in Porous Media, Dover, New York.

Bensoussan, A., J.-L. Lions, and G. Papanicolaou (1978), Asymptotic Analysis for Periodic Structures, Am. Math. Soc., North Holland.

Brun, E., and F. Touvier (1987), Etude expérimentale de la convection thermique dans la neige, J. Phys., 48(C1), 257-262.

Brun, E., E. Martin, V. Simon, C. Gendre, and C. Coléou (1989), An energy and mass model of snow cover suitable for operational avalanche forecasting, J. Glaciol., 35(121), 333-342.

Calonne, N., F. Flin, S. Morin, B. Lesaffre, S. Rolland du Roscoat, and C. Geindreau (2011), Numerical and experimental investigations of the effective thermal conductivity of snow, Geophys. Res. Lett., 38, L23501, doi:10.1029/2011GL049234.

Calonne, N., C. Geindreau, F. Flin, S. Morin, B. Lesaffre, S. Rolland du Roscoat, and P. Charrier (2012), 3-D image-based numerical computations of snow permeability: Links to specific surface area, density, and microstructural anisotropy, Cryosphere, 6(5), 939-951, doi:10.5194/tc-6-939-2012.

Calonne, N., C. Geindreau, and F. Flin (2014a), Macroscopic modeling for heat and water vapor transfer in dry snow by homogenization, J. Phys. Chem. B, 118(47), 13,393-13,403, doi:10.1021/jp5052535.

Calonne, N., F. Flin, C. Geindreau, B. Lesaffre, and S. Rolland du Roscoat (2014b), Study of a temperature gradient metamorphism of snow from 3-D images: Time evolution of microstructures, physical properties and their associated anisotropy, Cryosphere, 8(6), 2255-2274 doi:10.5194/tc-8-2255-2014

Colbeck, S. C. (1983), Theory of metamorphism of dry snow, J. Geophys. Res., 88(C9), 5475-5482, doi:10.1029/0JGREA0000880000C9005475000001.

Flin, F., and J.-B. Brzoska (2008), The temperature gradient metamorphism of snow: Vapour diffusion model and application to tomographic images, Ann. Glaciol., 49, 17-21, doi:10.3189/172756408787814834.

Fukuzawa, T., and E. Akitaya (1993), Depth-hoar crystal growth in the surface layer under high temperature gradient, Ann. Glaciol., 18, $39-45$

Geindreau, C., and J.-L. Auriault (2001), Transport phenomena in saturated porous media under liquid-solid phase change, Arch. Mech., 53, $385-420$.

Jordan, R. (1991), A one-dimensional temperature model for a snow cover: Technical documentation for SNTHERM.89, U.S. Army Cold Regions Research and Engineering Laboratory, Hanover, N. H.

Kaempfer, T. U., and M. Plapp (2009), Phase-field modeling of dry snow metamorphism, Phys. Rev. E, 79, 31,502, doi:10.1103/PhysRevE.79.031502.

Kamata, Y., and A. Sato (2007), Water-vapor transport in snow with high temperature gradient, in The Proceedings of the 11th International Conference on the Physics and Chemistry of Ice Held at Bremerhaven, Germany 23-28 July 2006, edited by W. Kuhs, pp. 281-288, Physics and Chemistry of Ice, RSC Publ., Cambridge, U. K.

Lasseux, D., A. A. Arani, and A. Ahmadi (2011), On the stationary macroscopic inertial effects for one phase flow in ordered and disordered porous media, Phys. Fluids, 23(7), 73,103, doi:10.1063/1.3615514.

Lehning, M., P. Bartelt, B. Brown, C. Fierz, and P. Satyawali (2002a), A physical SNOWPACK model for the Swiss avalanche warning. Part II: Snow microstructure, Cold Reg. Sci. Technol., 35(3), 147-167, doi:10.1016/S0165-232X(02)00073-3.

Lehning, M., P. Bartelt, B. Brown, and C. Fierz (2002b), A physical snowpack model for the swiss avalanche warning: Part III: Meteorological forcing, thin layer formation and evaluation, Cold Regions Sci. Technol., 35(3), 169-184.

Lehning, M., I. Völksch, D. Gustafsson, T. A. Nguyen, M. Stähli, and M. Zappa (2006), Alpine3D: A detailed model of mountain surface processes and its application to snow hydrology, Hydrol. Process., 20(10), 2111-2128, doi:10.1002/hyp.6204.

Massman, W. (1998), A review of the molecular diffusivities of $\mathrm{H}_{2} \mathrm{O}, \mathrm{CO}_{2}, \mathrm{CH}_{4}, \mathrm{CO}, \mathrm{O}_{3}, \mathrm{SO}_{2}, \mathrm{NH}_{3}, \mathrm{~N}_{2} \mathrm{O}, \mathrm{NO}$, and $\mathrm{NO}_{2}$ in air, $\mathrm{O}_{2}$ and $\mathrm{N}_{2}$ near STP, Atmospheric Environment, 32(6), 1111-1127, doi:10.1016/S1352-2310(97)00391-9. 
Mei, C. C., and J. L. Auriault (1991), The effect of inertia on flow through porous medium, J. Fluid. Mech., 222, 647-663. Neumann, T. A., M. R. Albert, C. Engel, Z. Courville, and F. Perron (2009), Sublimation rate and the mass-transfer coefficient for snow sublimation, Int. J. Heat Mass Transfer, 52(1), 309-315.

Powers, D., K. O'Neill, and S. C. Colbeck (1985), Theory of natural convection in snow, J. Geophys. Res., 90(D6), 10,641-10,649. Sanchez-Palencia, E. (1980), Non-homogeneous Media and Vibration Theory, Lectures Notes in Physics, vol. 127, Springer-Verlag, Berlin. Skjetne, E., and J.-L. Auriault (1999), High-velocity laminar and turbulent flow in porous media, Trans. Porous Media, 36(2), 131 -147. Sokratov, S., and A. Sato (2000), Wind propagation to snow observed in laboratory, Ann. Glaciol., 31(1), 427-433.

Sturm, M., and J. B. Johnson (1991), Natural convection in the subarctic snow cover, J. Geophys. Res., 96( B7), 11,657-11,671. Sturm, M., J. Holmgren, M. König, and K. Morris (1997), The thermal conductivity of seasonal snow, J. Glaciol., 43(143), $26-41$.

Vionnet, V., E. Brun, S. Morin, A. Boone, E. Martin, S. Faroux, P. L. Moigne, and J.-M. Willemet (2012), The detailed snowpack scheme Crocus and its implementation in SURFEX v7.2, Geosci. Model. Dev., 5, 773-791, doi:10.5194/gmd-5-773-2012.

Zermatten, E., M. Schneebeli, H. Arakawa, and A. Steinfeld (2014), Tomography-based determination of porosity, specific area and permeability of snow and comparison with measurements, Cold Reg. Sci. Technol., 97(0), 33-40, doi:10.1016/j.coldregions.2013.09.013. 